Research Article

\title{
Uncertainty Avoider Interval Type II Defuzzification Method
}

\author{
Sadegh Aminifar \\ Electrical Electronics Engineering Department, Mahabad Branch, Islamic Azad University, Mahabad, Iran \\ Correspondence should be addressed to Sadegh Aminifar; saminifar@yahoo.com
}

Received 18 January 2020; Revised 23 March 2020; Accepted 26 March 2020; Published 8 July 2020

Academic Editor: Jose Vicente Salcedo

Copyright $\odot 2020$ Sadegh Aminifar. This is an open access article distributed under the Creative Commons Attribution License, which permits unrestricted use, distribution, and reproduction in any medium, provided the original work is properly cited.

One of the IT2FS (interval type-2 fuzzy system) defuzzification methods uses the iterative KM algorithm. Because of the iterative nature of KM-type reduction, it may be a computational bottleneck for the real-time applications of IT2FSs. There are several other interval type-2 defuzzification methods suffering from lack of meaningful relationship between membership function uncertainties and changing of system output due to lack of clearly defined variables related to uncertainty in their methods. In this paper, a new approach for IT2FS defuzzification is presented by reconfiguring interval type-2 fuzzy sets and how uncertainties are present in them. This closed-formula method provides meaningful relation between the presence of uncertainty and its effect on system output. This study investigates uncertainty avoidance that the output of IT2FS obtained by centroid or bisection methods in comparison with type-1 fuzzy system (T1FLS) moves to points with less uncertainty. Uncertainty can enter into T1FSs and affect system response. Finally, for proving the affectivity of the proposed defuzzification method and uncertainty avoidance, several investigations are done and a prototype two-input one-output IT2FS MATLAB code is enclosed.

\section{Introduction}

In recent years, there has been an increasing trend of using type-II fuzzy systems (T2FSs) in engineering works. However, to use it practically, a number of obstacles have been faced, caused by lack of available methods appropriate for engineering applications, lack of clearly defined elements in systems, and lack of meaningful relationship between theoretical foundations and practical applications. In this section, some of the main IT2FM defuzzification methods are investigated in point of view of complexity and conceptuality.

There are various proposed defuzzification methods in literature such as the Karnik-Mendel iterative procedure (KMIP) [1] and the sampling defuzzification method [2] or direct defuzzifier [3]. None of these methods focused on the concept of uncertainty and its direct role in changing the system responses. Wu and Mendel [4] used uncertainty bounds for estimation of response, but it is based on mathematic interpretation and not on concept of uncertainty.

Defuzzifying methods presented by Begian et al. [5], Du and Ying [6], Nie and Tan [7], and John and Coupland [8] should not be categorized as fuzzy IT2 defuzzification because they are not capable to handle uncertainty in all conditions. It can be noted that they are not sensitive to imbalance of uncertainty. The proposed TR procedure in $\mathrm{Wu}$ and Tan [9] is applied before the inference engine, and it means that, in the first stage of an FLC, it finds an equivalent T1MF according to the inputs for fuzzification membership functions. Because of that proposed method in $\mathrm{Wu}$ and Tan [9], it can be considered as a method which finds an equivalent T1FLC with special input membership functions for its T2MF counterparts but not as a specific T2 defuzzification method. In Greenfield et al. [10], a T1MF between upper and lower bands was found as a representative embedded set, but the method and concept used are not clearly based on the concept of effect of uncertainty on certain data and membership degrees. The efficiency, functionality (applicability), simplicity, and conceptuality are also better than Greenfield et al. [10].

$\mathrm{Wu}$ and Mendel [11] pointed out that the KM algorithms are not always convergent. It should be noted that when all sources are free of uncertainty, the KM algorithm is reduced to T1FS. However, as $\mathrm{Wu}[12]$ indicated, the (type reduction) TRKM approach is not the only way to achieve the design 
requirements. In fact, other alternative methods have been proposed. For example, Nie and Tan [7] defined four other types for TR, namely, optimistic, pessimistic, realistic, and weighted realistic. John and Coupland [8] have proposed a geometric representation and an approach based on GT2FS operations. Real-world application of the work of Copland and John shows that the response of the system is faster in comparison with the KM reduction method.

To reduce the computational complexity of KM algorithms, Wu and Mendel [4] extended the IT2FS uncertainty bounds to estimate TR. They showed that the uncertainty bounds can be defined with no need to TR, while we will obtain results similar to that achieved by the KM algorithm. Thus, it was suggested that this method can be used to design IT2FLS. The main problem with this method is that the calculations and the defined parameters lack a proper interpretation. In contrast, the hardware implementation as a CMOS chip is hardly possible for proposed formulas. There are many obstacles for hardware-based implementation of interval and general T2FSs. Transistor level T1FSs are usually complex [13]; many code-based applications are reported in the literature for T2FSs [14-16].

$\mathrm{Wu}$ and Mendel [11] developed an improved algorithm called enhanced Karnik-Mendel (EKM) algorithm. They showed that this algorithm reduces the computation time more than 39\%. Celemin and Melgarejo [17] proposed a recursive algorithm to compute the center of mass for T2MFs. The new algorithm specifies the constraints in T2MF center of mass. The performance of this algorithm is evaluated by a numerical analysis. The results showed that this method is considerably faster than the KM algorithm for the symmetric footprint of uncertainty (FOU) and for producing similar results. However, this method is yet to use as an algorithm and there is no hardware proof of its performance.

While the KM algorithm is widely used in software, some new inference engines have been proposed to circumvent the TR. For example, Wu and Mendel [18] introduced a method in which the equivalent $\mathrm{T} 2$ set is replaced by a T1 set. It was shown that this method performs better than an iterative $\mathrm{KM}$ algorithm. However, the mentioned method is provided for a two-input PI controller and it is not known that whether it can be extended to more complex systems or not.

Runkler et al. [19] proposed a defuzzification method considering uncertainty weights. The most similar idea to Runkler's method named UW is [20]. UW has two problems regarding uncertainty handling which is discussed in Section 4 of this paper (investigation 7).

The author of this paper investigated uncertainty effect in IT2FSs to overcome the abovementioned problems in [20]. The present research is mainly a better description of [20], which extends to a specific defuzzification method based on uncertainty effect called the uncertainty avoider method. The complexity of defuzzification calculations in general T2FSs is more than IT2FSs. A method is presented in [21] for reduction of complexity cost in general T2FS based on parametrized shadowed type-II membership functions. Ontiveros-Robles et al. [22, 23] also worked on decreasing the computational cost of general T2FS defuzzification. The extension of uncertainty avoider method to the general typeII fuzzy systems is found in [24].

\section{Methodology}

2.1. Rule Base and Control Surface. A set of rules, which define the behaviour of a system, assumes the description of an extensive set of control surfaces [25]. Moreover, none of these control surfaces are applicable to the proposed pertinent system, and in fact, only one of these control surfaces is considered and fuzzy system manages to find it. It is better to say that the systems, which should only be controlled by a certain control surface, principally will have a weak position in computing with words (fuzzy systems) [26].

\subsection{Main Criteria for Choosing Defuzzification Methods.} Defuzzifying method is responsible for limiting the atmosphere described by rule base. Different defuzzification methods lead to different responses. Once it is said that one defuzzification method is more coordinated with lexical calculation than the other methods; when using that method, it would be more probable to estimate the output based on the descriptive understanding of system behaviour for designers. In addition, a system is considered as a CW (computing with words) system when designers have more conceptual tools to be able to create more useful changes in a system based on the descriptive information. Li Xin Wang, "A course in fuzzy systems and control," Prentice Hall International, 1997, page 108, cited three criteria for defining defuzzification methods:

2.3. Plausibility. The defuzzified output of a defuzzification method should represent membership function from an intuitive point of view; for example, it may lie approximately in the middle of the support of corresponding MF or has a high degree of membership in MF. Computational simplicity is particularly important for fuzzy control because fuzzy controllers operate in real-time. Continuity is a small change in MF that should not result in a large change in defuzzified output.

For example, "A course in fuzzy systems and control" (page 109) explains that the only advantage of the center of gravity (COG) defuzzifier lies in its intuitive plausibility. And the main disadvantage of COG is that it is computationally intensive.

This paper adds the fourth criterion especially for IT2FS defuzzification. Conceptuality is changing the parameters and variables that are used to calculate defuzzified output which should have meaningful relation with output changes.

This research proposes a simple close-formula defuzzification method for IT2FSs which satisfies all four aforementioned criteria for defuzzification definition. Rest of this section is dedicated to describe our aim in more details and provide the necessary definitions.

In this paper, uncertainty is the uncertainty of membership degree of members of a fuzzy set. Figure 1 shows our interpretation of IT2MF as an extension of type- 1 fuzzifier. The fuzzy system proposed in this research uses a readout of 


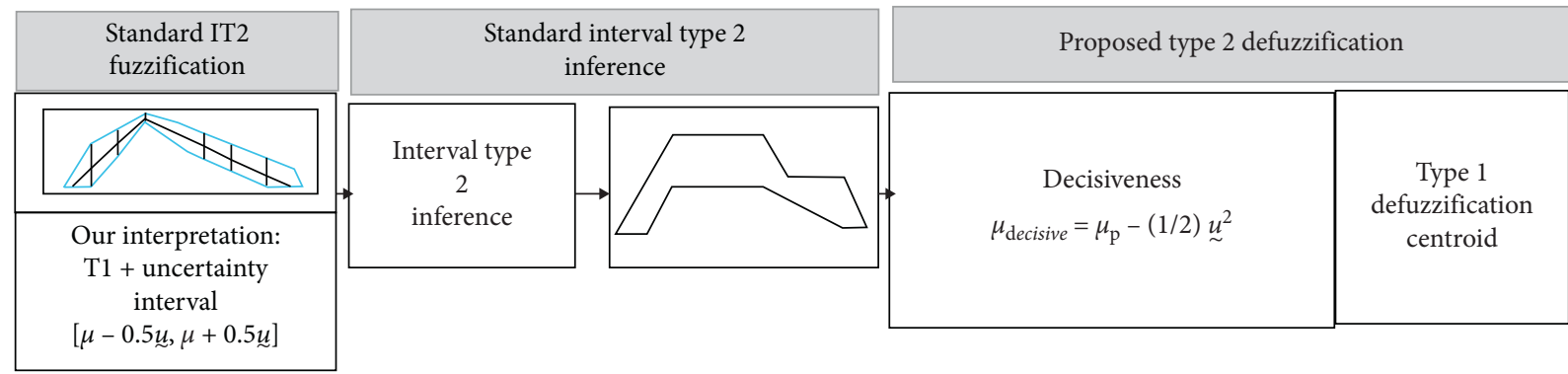

FIgURE 1: Proposed IT2FS blocks.

IT2FSs that defines IT2FS as an extension of T1FSs. Inference engine normally works like standard IT2FS. In last stage of IT2FS as shown in Figure 1, uncertainty remover (decisiveness block) is added before type-1 defuzzification, which is the main contribution of this research.

2.4. Interval Type-2 Fuzzifier Interpretation. The IT2MF is considered as T1MF in which each membership degree is uncertaintified by adding equal amount of uncertainty to its upper and lower sides. Figure 2 shows our definition of uncertainty band and an IT2MF and its counterpart T1MF.

UMF: upper bound of uncertainty; $\mu_{\mathrm{u}}=$ upper membership degree of each member of support of IT2MF.

LMF: lower bound of uncertainty; $\mu_{1}=$ lower membership degree of each member of support of IT2MF. PMF: principal membership function which is the average of UMF and LMF mathematically.

$\mu_{\mathrm{p}}$ : principal membership degree of each member of support of IT2MF. The relation between $\mu_{\mathrm{p}}, \mu_{\mathrm{u}}$, and $\mu_{1}$ is shown as follows:

$$
\mu_{\mathrm{p}}=\frac{\mu_{u}+\mu_{l}}{2}, \quad 0 \leq \mu_{\mathrm{p}} \leq 0.5
$$

Uncertainty band function $(\underset{\sim}{U})$ : for each IT2MF, one defines a function, which shows the uncertainty band for each point (member) of support of IT2MF.

$\underset{\mathcal{u}}{u}=$ uncertainty band degree; interval height (considering that the membership degree of IT2MF is an interval): it is defined as the difference between upper and lower membership degrees:

$$
\underline{u}=\mu_{\mathrm{u}}-\mu_{\mathrm{l}}, \quad 0 \leq \underset{\sim}{\mathcal{u}} \leq 1
$$

Considering equations (1)-(3) is concluded:

$$
\begin{aligned}
& \mu_{\mathrm{u}}=\mu_{\mathrm{p}}+\frac{1}{2} \underset{\sim}{\underline{u}}, \\
& \mu_{\mathrm{l}}=\mu_{\mathrm{p}}-\frac{1}{2} \underset{\sim}{\sim} .
\end{aligned}
$$

Equation (3) clarifies our interpretation of IT2MF. Each IT2MF is an extension of a type-1MF in which by increasing and decreasing specific amount of uncertainty $0.5 \underset{\sim}{\mathrm{u}}$, upper and lower bounds are created.

Using $\underset{\sim}{u}=\mu_{\mathrm{u}}-\mu_{\mathrm{l}}, I$ defined a new parameter $U$ to use it as a parameter to change $\underset{\sim}{\mathcal{u}}$ proportional to $\underset{\sim}{\mathcal{u}}$. It can be defined as ${\underset{\mathcal{u}}{2}}^{2}$. On the contrary, as it is known from equation (1), the $\mu_{\mathrm{p}}$ is $\left(\mu_{\mathrm{u}}+\mu_{\mathrm{l}}\right) / 2$ and it varies between 0 and 0.5 . Because of that, it is expected to compare $U$ with $\mu_{\mathrm{p}}$. Therefore, $U$ is defined as $(1 / 2){\underset{\sim}{u}}^{2}$ instead of ${\underset{\sim}{u}}^{2}$. $U$ is named the uncertainty portion:

$$
U=\frac{1}{2} \underset{\sim}{2} \stackrel{2}{\sim}=\frac{1}{2}\left(\mu_{\mathrm{u}}-\mu_{1}\right)^{2}, \quad 0 \leq U \leq 0.5
$$

One feature of $U$ is that, if uncertainty $\underset{\sim}{u}$ is low, $U$ is very low. And if uncertainty $\underset{\sim}{u}$ is high, $U$ is very high. For example, if $\mu_{\mathrm{p}}$ is $\mathrm{T} 1$ and it is certain, uncertainty band is zero (i.e., $\underset{\sim}{u}=0$ ), then the portion of uncertainty is zero. But if $\mu_{\mathrm{p}}$ is completely uncertain, uncertainty band is in the highest state (i.e., $\underset{\sim}{u}=1$ ), and then the portion of uncertainty is 0.5 , which is highest amount for $U$.

2.5. Decisiveness Definition. If a $[a b]$ interval variable in any system is replaced by " $c$ " $(a \leq c \leq b)$ number (as shown in Figure 3) in a way that the system's previous behaviour (output) is not changed or new desirable behaviour appear, point " $c$ " is called as the decisive point of $[a b]$ interval. It means point " $c$ " makes decision instead of interval $[a b]$ and plays as an absolute representative of interval $[a b]$. If all intervals are determined to their corresponding decisive point, a membership function appeared that it is called decisive membership function (DMF).

\section{Proposed Defuzzifier and Its Property}

For each interval of IT2MF, one decisive membership degree is defined. The proposed formula is based on this fact; the more uncertainty band around $\mu_{\mathrm{p}}$ causes more decrease of membership of $\mu_{\mathrm{p}}$. And certain $\mu_{\mathrm{p}}$ should not change. Therefore, uncertainty portion is used as in equation (5) to modify the membership degree of $\mu_{\mathrm{p}}$ proportion to uncertainty portion $U$ :

$$
\mu_{\text {decisive }}=\mu_{\mathrm{p}}-U .
$$

First, each uncertainty band corresponding to each point of principal function is reduced to one certain point using the below decisiveness formula, and hereafter, the arbitrary type-one defuzzification is applied: 


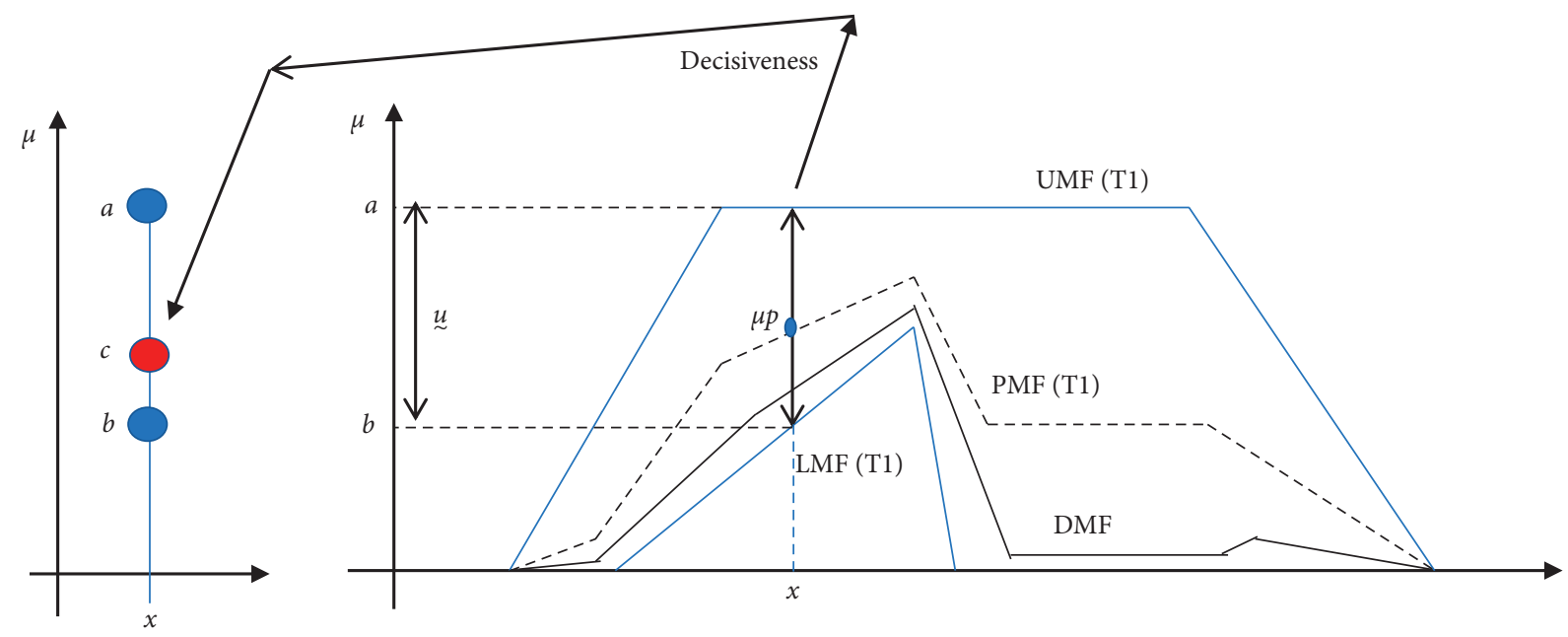

Figure 2: IT2FM and UMF, LMF, PMF, $\underset{\sim}{\mathrm{u}}, \mu_{\mathrm{u}}, \mu_{\mathrm{l}}$, and $\mu_{\mathrm{p}}$.

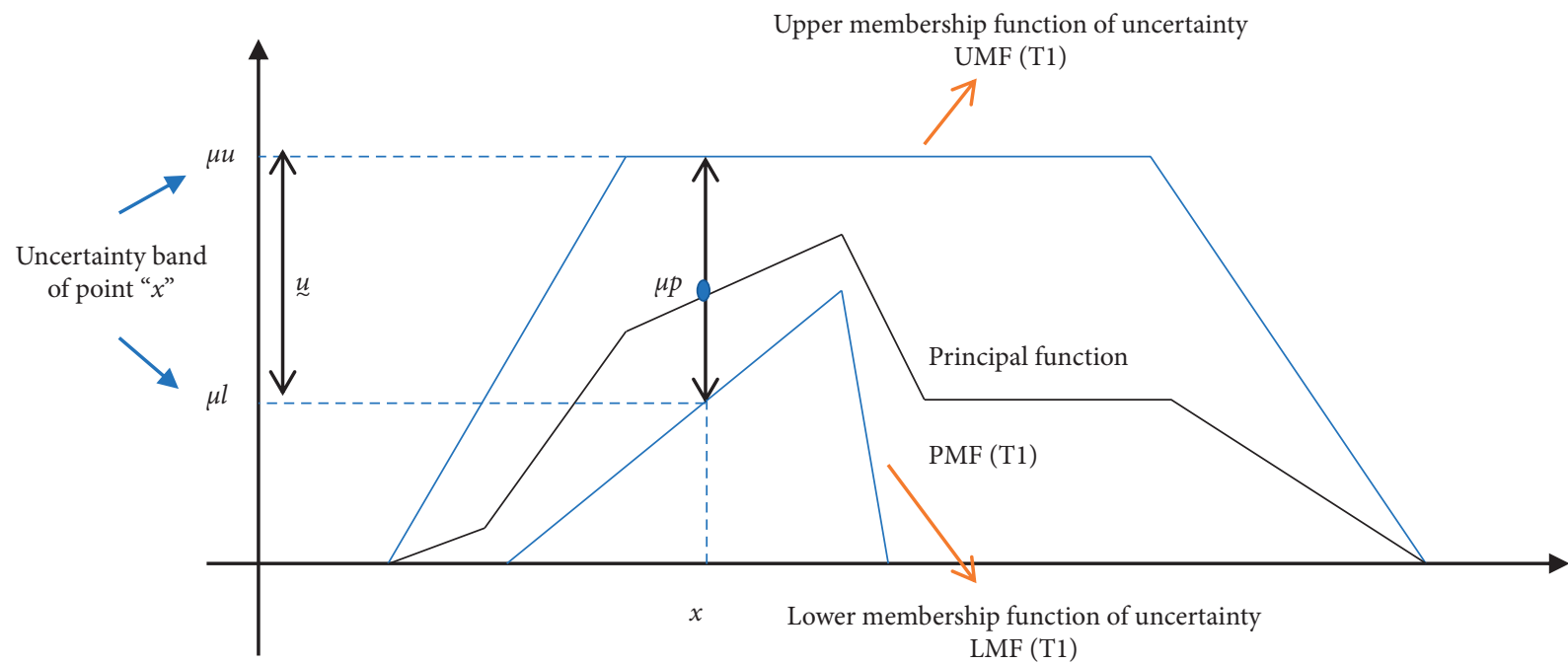

Figure 3: Decisiveness and DMF.

$$
\mu_{\text {decisive }}=\mu_{\mathrm{p}}-\left(\frac{1}{2}\right) \underset{\sim}{\underline{u}^{2}} .
$$

One of the advantages of this decisiveness formula is that the more the uncertainty band increases, the decisive value of a point decreases proportionally. On the contrary, this equation guaranties by varying $\underset{\sim}{\boldsymbol{u}}$ between its minimum and maximum amounts (i.e., 0 and 1 ); the determined membership degree stayed in its range (i.e., [0 1]).For instance, if $\underline{u}=1$, then $\mu_{\mathrm{dtr}}=0$; if $\underset{\mathcal{u}}{\mathcal{u}}=0$, then $\mu_{\mathrm{dtr}}=1$, and its fluctuations between these two points are in accordance with $\underset{\sim}{u}$. There is a concept behind this equation. It shows higher uncertainty involved with a member (i.e., longer interval membership degree) causes a higher loss of membership degree of corresponding member.

3.1. Uncertainty Avoidance Definition. In proportion to the centroid of the principal MF, the centroid of IT2FM is displaced toward a more certain side. A higher amount of uncertainty imbalance on both sides of the centroid of the principle MF corresponds to more displacement that shifts the new centroid toward certain side.

For example, as shown in Figure 4, if some uncertainty is injected to one side of the COG of IT2MF, the COG will shift toward the other side. An increasing uncertainty on the left of Points $b$ and $c$ displaces the response (COG) toward the right compared with Point $a$ (Figure 4). In Points $c$ and $d$, creating a slight uncertainty toward the right slightly displaces the response (COG) toward the left. The shadow around each point shows the uncertainty around that point (Figure 4).

3.1.1. Uncertainty Avoidance and Area Bisection Defuzzification. In this section, one aspect of uncertainty avoidance of applying equation (6) before bisection defuzzification is verified. It is shown the imbalance of the 


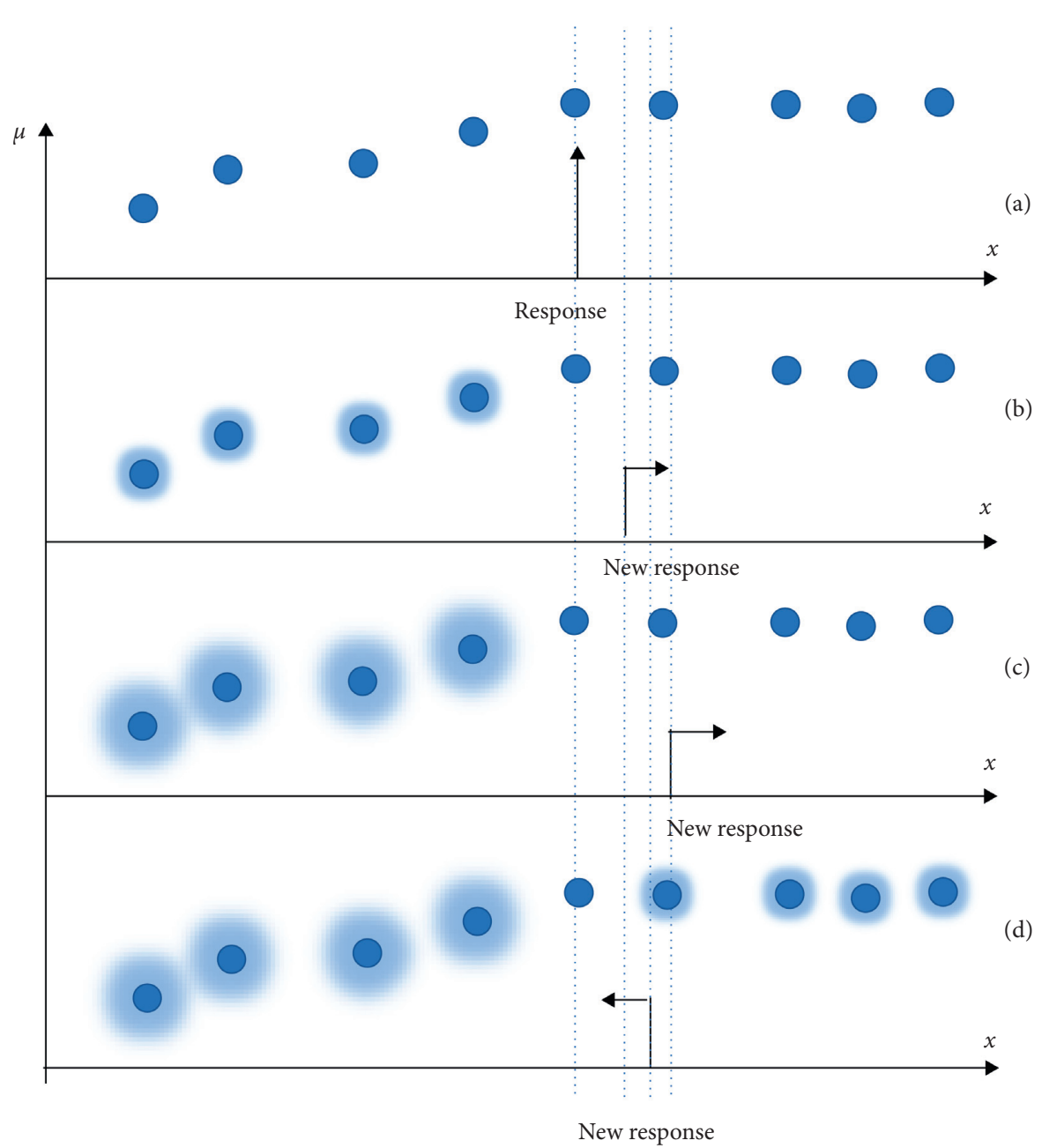

Figure 4: (a) Both sides are certain (T1); (b) left side is slightly uncertain; (c) left side is more uncertain; (d) additional uncertainty to the right side of (c).

uncertainty directly and proportionally displaces the center achieved by the bisection defuzzification method.

(1) Bisection (BS) Defuzzification. This method divides the membership function in two equal area parts, as shown in Figure 5(a). For discrete MFs, it divides the MF to two parts with equal powers; i.e., a point is found in support of MF to satisfy

$$
\sum_{\text {left }} \mu=\sum_{\text {right }} \mu
$$

For discrete MFs as shown in Figure 5(b), BS defuzzification is achieved by finding center in

$$
\int_{\text {Start }}^{\text {Center }} \mu \mathrm{d} y=\int_{\text {center }}^{\text {End }} \mu \mathrm{d} y \text {. }
$$

Considering Figure 5(b), there is a center which satisfies $\sum_{\text {left of center }} \mu=\sum_{\text {right of center }} \mu$.

That is,

$$
\mu_{0}+\mu_{1}+\mu_{2}+\cdots+\mu_{\mathrm{x}}+\cdots=\mu_{\mathrm{a}}+\mu_{\mathrm{b}}+\mu_{\mathrm{c}}+\cdots+\mu_{\mathrm{f}}+\cdots .
$$

Some members in left side uncertaintified (IT2MF), as shown in Figure 6.

Applying equation (6) to remove uncertainty of left side ( $\mu_{\mathrm{x}}$ replaced with $\left(\mu_{\mathrm{x}}-U_{\mathrm{x}}\right)$ ( $U$ is uncertainty portion, equation (4)),

$$
\mu_{0}+\mu_{1}+\mu_{2}+\cdots+\mu_{\mathrm{x}}-U_{\mathrm{x}}+\cdots<\mu_{\mathrm{a}}+\mu_{\mathrm{b}}+\mu_{\mathrm{c}}+\cdots+\mu_{\mathrm{f}}+\cdots,
$$

and for balancing (10), the center should move to the right side to include some members of the right side in such way that power of both sides of new center becomes equal. It is clear that if more members of the left side become uncertain (IT2MF), the new center must move more to the opposite side (right side) to make balance between the power of both sides new membership degrees.

In continuous state, the center line, as shown in Figure 5(a), made both sides in balance in point of view of area. It is clear the presence of uncertainty in left side causes area loss. Therefore, it is completely logical to displace new center to the right side for compensation the area loss. 


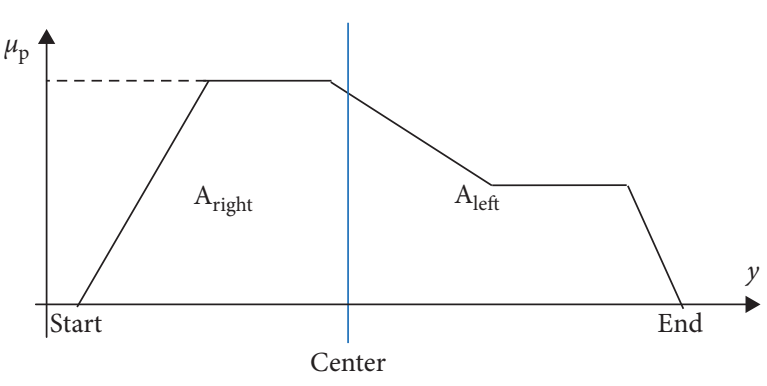

(a)

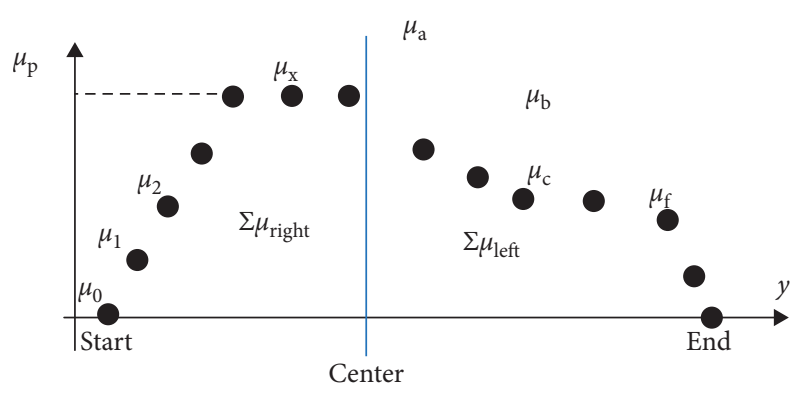

(b)

Figure 5: (a) Continuous T1MF and its bisection center; (b) discrete T1MF and its bisection center.

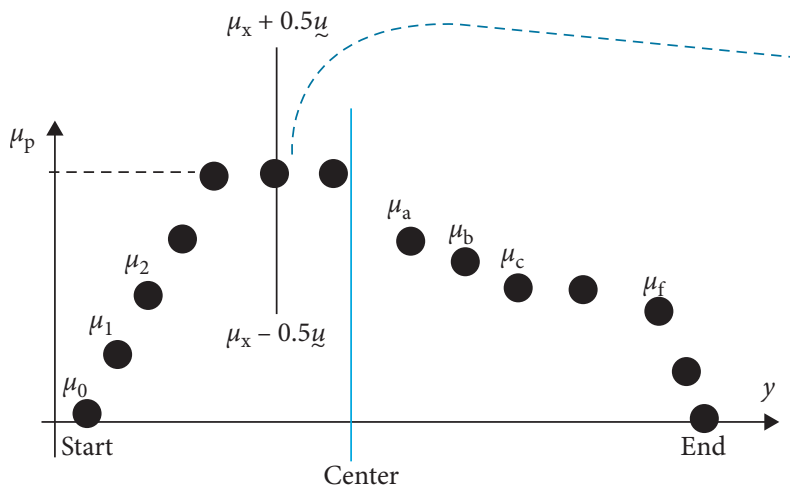

(a)

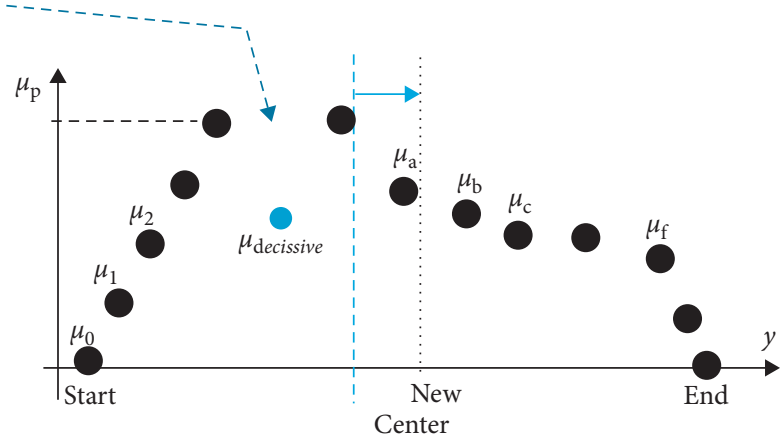

(b)

Figure 6: (a) Discrete T1MF (certain membership degrees) which by uncertain of membership $\mu_{x}$ converted to IT2MF; (b) discrete decisive MF (DMF) using equation (6).

3.1.2. Decisiveness Method and COG Defuzzification. In this section, it is shown that, in centroid defuzzification, the displacement of centroid depends on the imbalance between the power of uncertainty of each side and the moment of uncertainty portion of each side, simultaneously. The similarity of effectiveness of moment of uncertainty to moment of membership degree which is the nature of finding COG is interesting. Using equation (6) before T1MF COG confirms uncertainty avoidance.

The centroid defuzzification method used in fuzzy systems originated from mass centroid concept to find the centroid of a homogenous piece of a material. The center of a mass is a point that if the body is supported at its centroid point, it balances perfectly. In other words, in Figure 7, the moment (torque) of both sides of the centroid must be equal. The centroid upon its definition in physics obeys equation (11).

Total moment of the left side of the centroid = total moment of the right side of the centroid:

$$
m_{1} d_{1}+m_{2} d_{2}=m_{3} d_{3}+m_{4} d_{4}
$$

where $d_{1}, d_{2}, d_{3}$, and $d_{4}$ are horizontal distance of $m_{1}, m_{2}, m_{3}$, and $m_{4}$ to COG, respectively.

Generally, $\quad \sum_{1}^{\text {Centroid }}$ midi $=\sum_{\text {centroid }}^{m} m j d j$, and for continuous state, $\int_{0}^{\text {centroid }} m(d) d d=\int_{\text {centroid }}^{\text {end }} m(d) d d$.

Considering below MF, we repeat general equation of centroid using $\mu$ instead of $m$ :

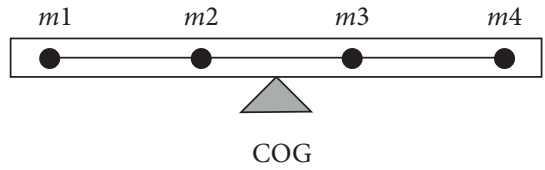

FIgURE 7: The concept of mass centroid.

$$
\sum_{1}^{\text {Centroid }} \mu i d i=\sum_{\text {centroid }}^{m} \mu j d j .
$$

Now, by adding uncertainty to a member of the left side of the centroid in Figure 8 and applying decisiveness formula, due to decreasing $\mu_{\mathrm{x}}$ to its new amount $\mu_{\text {decisive, we will have }}$

$$
\sum_{\text {Start }}^{\text {oldCentroid }}(N e w \mu i) d i<\sum_{\text {Old Centroid }}^{\text {End }} \mu j d j .
$$

Therefore, new centroid must move to right side for satisfying balance of both sides of (13). Then, it is concluded that, by adding uncertainty to the left side, the centroid shifted to the right side. This shows that the decisiveness equation satisfies uncertainty avoidance.

Now, if it is considered that the movement of new centroid to the right is $d_{\mathrm{r}}$, writing centroid equation, we have 


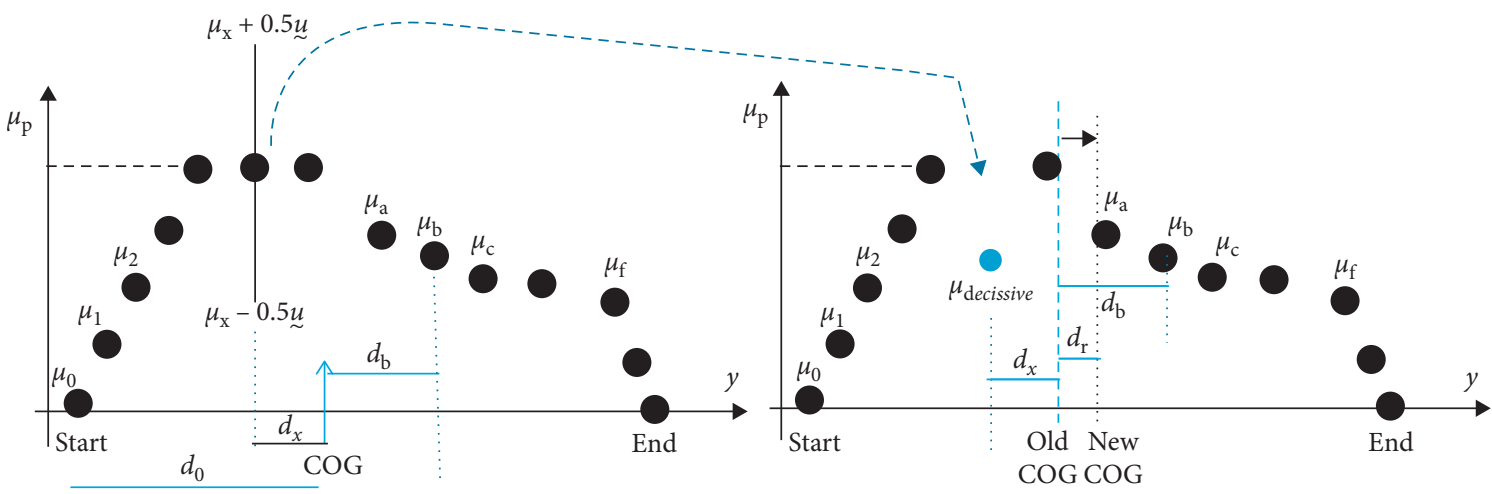

(a)

(b)

FIGURE 8: (a) COG of PMF (T1MF) before presence of uncertainty; (b) COG after applying equation (6).

$$
\begin{aligned}
& \mu_{0}\left(d_{0}+d_{\mathrm{r}}\right)+\mu_{1}\left(d_{1}+d_{\mathrm{r}}\right)+\mu_{2}\left(d_{2}+d_{\mathrm{r}}\right)+\cdots+\mu_{\text {xnew }}\left(d_{x}+d_{\mathrm{r}}\right)+\cdots \\
& =\mu_{\mathrm{a}}\left(d_{\mathrm{a}}-d_{\mathrm{r}}\right)+\mu_{\mathrm{b}}\left(d_{\mathrm{b}}-d_{\mathrm{r}}\right)+\mu_{\mathrm{c}}\left(d_{\mathrm{c}}-d_{\mathrm{r}}\right)+\cdots+\mu_{\mathrm{f}}\left(d_{\mathrm{f}}-d_{\mathrm{r}}\right)+\cdots,
\end{aligned}
$$

where $d_{0}, d_{1}, d_{2}, \ldots, d_{\mathrm{x}}, \ldots, d_{\mathrm{a}}, d_{\mathrm{b}}, d_{\mathrm{c}}, \ldots$ and $d_{\mathrm{f}}$ are horizontal distances of $\mu_{0}, \mu_{1,}, \mu_{2}, \ldots, \mu_{\mathrm{x}}, \ldots, \mu_{\mathrm{a}}, \mu_{\mathrm{b}}, \mu_{\mathrm{c}}, \ldots$ and $\mu_{\mathrm{f}}$, respectively. As an example, $d_{0}, d_{\mathrm{x}}$, and $d_{\mathrm{b}}$ are shown in Figure 8. $d_{\mathrm{r}}$ is the displacement to right, as shown in Figure 8.

Note: for writing equation (14), it is considered that displacement of centroid to right side is lesser than including the previous left side members. But if we consider that displacement occupies some members of old right side, it does not affect the procedure of simplifying this equation to reach the conclusion (i.e., equation (17)).

Considering equation (6) and replacing $\mu_{\mathrm{x}}$ with $\mu_{\text {xnew }}=\mu_{\mathrm{x}}-U(U$ is the uncertainty portion, Equation (4)), then

$$
\begin{aligned}
& \mu_{0} d_{0}+\mu_{1} d_{1}+\mu_{2} d_{2}+\cdots+\mu_{\mathrm{x}} d_{\mathrm{x}}-U d_{\mathrm{x}}+\cdots \\
& \quad+\left(\mu_{0}+\mu_{1}+\mu_{2}+\cdots+\mu_{\mathrm{x}}+\cdots\right) d_{\mathrm{r}}-U d_{\mathrm{r}} \\
& =\mu_{\mathrm{a}} d_{\mathrm{a}}+\mu_{\mathrm{b}} d_{\mathrm{b}}+\mu_{\mathrm{c}} d_{\mathrm{c}}+\cdots+\mu_{\mathrm{f}} d_{\mathrm{f}}+\cdots \\
& \quad-\left(\mu_{\mathrm{a}}+\mu_{\mathrm{b}}+\mu_{\mathrm{c}}+\cdots+\mu_{\mathrm{f}}+\cdots\right) d_{\mathrm{r}}
\end{aligned}
$$

Then,

$$
\begin{gathered}
\left(\sum_{\text {Start }}^{\text {old Centroid }} \mu i d i\right)+\mathrm{d} r \sum \mu(\text { left side })-U \mathrm{~d} x-U \mathrm{~d} r \\
\left.=\left(\sum_{\text {old Centroid }}^{\text {End }} \mu j d j\right)-\mathrm{d} r \sum \mu \text { (right side }\right) .
\end{gathered}
$$

\footnotetext{
Considering equation $(14), \quad\left(\sum_{\text {Start }}^{\text {old Centroid }} \mu i d i\right)=$ $\left(\sum_{\text {old Centroid }}^{\text {End }} \mu j j\right)$, then
}

$\mathrm{d} r \sum \mu$ (left side) $-U \mathrm{~d} x-U \mathrm{~d} r=-\mathrm{d} r \sum \mu$ (right side),

$\mathrm{d} r \sum \mu($ left side $)-U \mathrm{~d} r+\mathrm{d} r \sum \mu($ right side $)=U \mathrm{~d} x$,

$$
\begin{aligned}
& \mathrm{d} r=\frac{(U d x)}{\left(\sum \mu(\text { left side })+\sum \mu(\text { right side })-U\right)}, \\
& \mathrm{d} r=\frac{(U \mathrm{~d} x)}{\left(\sum \mu(\text { left side }+ \text { right side })\right)}-U, \\
& \mathrm{~d} r=\frac{(U d x)}{\left(\left(\sum \mu\right)-U\right)} .
\end{aligned}
$$

If there are more points in left side with uncertainty, simply, we can extend the above equation to

$$
\begin{aligned}
\mathrm{d} r & =\frac{\sum_{\mathrm{left}} U \times d}{\sum \mu-\sum_{\mathrm{left}} U} \\
& =\frac{\text { total left side uncertainty portion moment }}{\text { MF power }- \text { left side uncertainty portion power }} .
\end{aligned}
$$

This equation is simply applicable for $d_{1}$ (displacement of centroid to the left side in the presence of uncertainty in right side only):

$\mathrm{d} l=\frac{\sum_{\text {right }} U \times d}{\sum \mu-\sum_{\text {right }} U}$

$$
=\frac{\text { total right side uncertainty portion moment }}{\text { MF power }- \text { right side uncertainty portion power }} \text {. }
$$


Considering superposition law due to linear nature of equation (6), $d$ is the displacement of centroid in presence of uncertainty in both sides of COG of PMF:

$$
d=\mathrm{d} r-\mathrm{d} l=\frac{\sum_{\mathrm{right}}(U \times d)}{\sum \mu-\sum_{\mathrm{right}} U}-\frac{\sum_{\mathrm{left}}(U \times d)}{\sum \mu-\sum_{\mathrm{left}} U} .
$$

This equation shows that the displacement depends on imbalance of uncertainty. The interpretation of uncertainty imbalance according to equation (20) is the imbalance between the power of uncertainty of each side and the moment of uncertainty of each side, simultaneously. If the uncertainty of both sides of the centroid is equal, then the displacement directly depends on the imbalance of the uncertainty moment. " $d_{\mathrm{r}}$ " and " $d_{1}$ " are positive because always $\sum \mu>\sum_{\text {right }} U$ and $\sum \mu>\sum_{\text {left }} U$.

If $d>0$, total centroid displacement is toward the right side, and if $d<0$, displacement is toward the left side.

\section{Investigations and Discussion}

4.1. Investigations. These numerical investigations intend to show a comparative behaviour of the proposed defuzzification method. Investigation 1 just wants to show the illustrative concept of equation (6). Investigation 2 shows comparative outputs as a result of applying the proposed defuzzification method and the KM algorithm of 32 basic IT2MF words. A single-input single-output fuzzy system was introduced in investigation 3 as a base to compare the output created by the proposed method and by different approaches clearly. In investigation 4 , the effects of different types of uncertainty in system output are investigated. Investigation 5 discusses the uncertainty avoidance in noisy data. In investigation 6 , a numerical comparison between the collapsing method [10] and proposed work is done. In investigation 7, a comparison between UW method [19] and determiners proposed in [20] is done. Investigation 8 shows the uncertainty effect on a two-input one-output IT2FS and presents a general MATLAB code for IT2FSs based on the uncertainty avoidance method in the defuzzification stage.

Examples of investigations are chosen in such a way to cover the generalities of the issue, by selecting various forms of membership functions and changing the different statuses of antecedent and subsequent membership functions in a way to create various MFs. The root of the mean of squared (RMS) of differences and the maximum difference are shown in different statuses. The common form of the trapezoidal and triangular MF can be described by a five-number vector $[a, b, c, d$, and $e]$ (Figure 9).

Investigation 1: Observing Decisive Membership Function (DMF) Illustrations

(1) For an IT2MF $(\mathrm{UMF}=[0,0,0.64,2.63,1]$ and $\mathrm{LMF}=$ $[0,0,0.09,0.99,1])$, calculate the following:

(a) Output defuzzified value using DMF (decisive membership function achieved by equation (6))

(b) Calculate the difference percentage between obtained defuzzified value using DMF and COG of mentioned IT2MF based on the KM algorithm

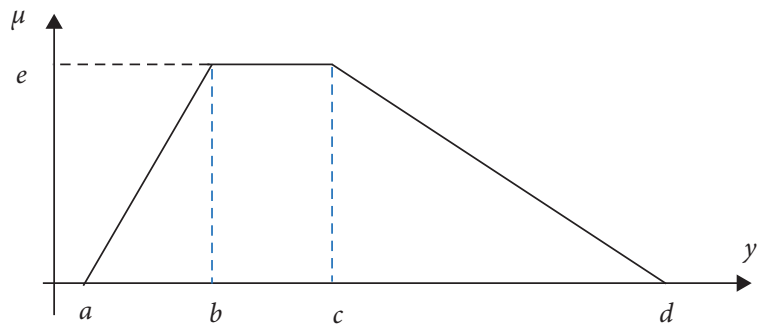

FIgURE 9: Common form of trapezoidal MF shown by five-number vector $[a, b, c, d, \mathrm{e}]$.

Answer: COG of PMF shown in Figure 10 is COG $(\mathrm{PMF})=0.7694$, using decisive DMF obtained by equation (6), and the shown decisive membership function in Figure 10 is obtained, while its COG is easily calculable as COG (decisive $)=0.6241$.

COG of shown IT2MF in Figure 10 using the KM algorithm is 0.69 , and for calculating the difference of these two COGs, equation (21) is used:

$$
\mathrm{E}=\frac{\mid \mathrm{COG}(\text { decisive })-\mathrm{COG}(\mathrm{KM}) \mid}{\text { support of UMF of IT2MF }} \times 100 \% \text {. }
$$

(2) Repeat calculations for $\mathrm{UMF}=[5.98,7.75,8.60,9.52$, 1] and $\mathrm{LMF}=[8.03,8.36,8.36,9.17,0.57]$ and calculate COG based on DMF.

Here, $E=2.5 \%$.

Answer: COG of PMF shown in Figure 11 is COG $(\mathrm{PMF})=7.9966$ and COG $(\mathrm{DMF})=8.1864$. UMF, LMF, PMF, and DMF (using equation (6)) are illustrated in Figure 11.

The difference percentages between these COGs are 0.5\% and calculated using equation (21).

Investigation 2: A Numerical Comparison between KMBased COG and Decisive COG

$\mathrm{Wu}$ and Mendel showed in [11] that the defuzzified output of 32 basic interval fuzzy T2 words is based on the $\mathrm{KM}$ algorithm. In this research, their defuzzified output is calculated by using the proposed defuzzification method.

Table 1 shows those words using the 5 parameters for introducing trapezoidal as in Figure 9. Equation (21) is used for calculation of difference percentage between KM-based COG and proposed method. In case of slight output differences between this method and the KM algorithm, no proof exists that shows that the outputs gained by the KM method are generally better than other methods. On the contrary, the existence of concepts and reasons behind our proposed method provides a designer with more opportunities to manage parameters related to uncertainty in IT2 fuzzy controllers in engineering and industrial affairs conveniently.

Investigation 3: Using Decisiveness Method for Describing the Impact of Uncertaintified Output Fuzzy Words on the Output of a SISO FLC

The specifications of a given single-input single-output single-rule system (as shown in Figure 12) are as below. 


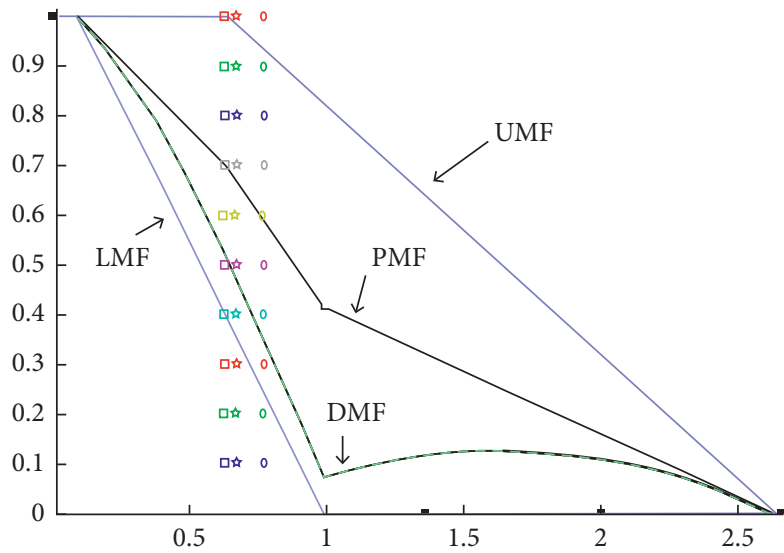

Figure 10: IT2MF and its PMF and decisive MF (PMF COG shown by "o" is 0.7694 ; KM COG shown by "star" is 0.69 ; decisive COG shown by "square" is 0.6241 ).

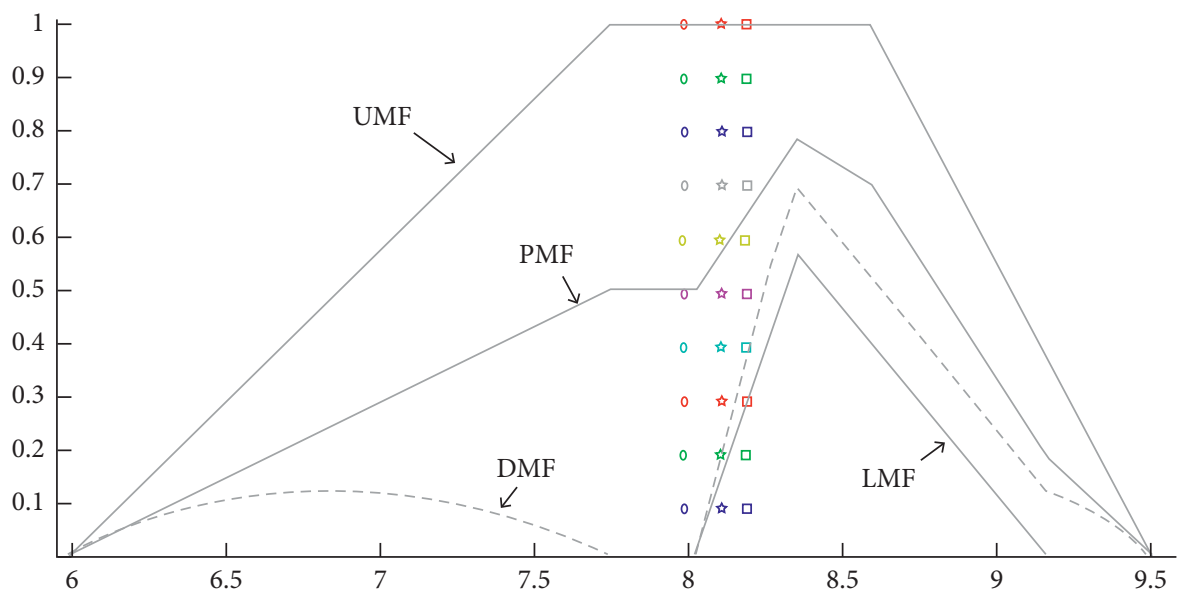

FIGURE 11: IT2MF and its PMF and DMF (PMF COG is shown by "o;" KM COG is shown by "star;" decisive COG is shown by "square").

The calculations and illustrations regarding control surface (or system mapping curve) for both uncertaintified output fuzzy words are shown in Figures 13(a) and 13(c).

As shown in Figures 13(b) and 13(d), the output is affected by adding uncertainty to fuzzy output T1MFs. Figures 13(b) and 13(d) show that the uncertainty makes the rules to be weakened with respect to the total injected uncertainty to output fuzzy MF (not to each point). In this case, the rule is "if input is small then output is small," but if the effect of uncertainty is considered in Figure 13(b), it can be seen that the response after applying the uncertainty is greater than the system with T1MF input. Figure 13(d) shows the increasing injected uncertainty causes the increasing system output, and the related rule is more weakened than before. It shows the concept of "small" in output to some extend is shifted towards the concept of "medium" or "large." This investigation shows that the uncertainties output fuzzy word is considered as a new unique word which describes more complex word than T1 fuzzy word.

Investigation 4: Using Decisiveness Method for Describing the Impact of Uncertaintified Input Fuzzy Words on Output of a SISO FLC
The specifications of a single-input single-output singlerule system (as shown in Figure 12) are as follows:

Rule: if $X$ is small, then $Y$ is small

Implication $=$ Min

$Y$ (output) T1MF: small (trapezoidal MF) $=\left[\begin{array}{lllll}0 & 0 & 0 & 1 & 1\end{array}\right]$

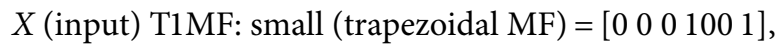
which is uncertain, as shown in Figure 14(a).

The calculations and illustrations regarding control surface (or system mapping curve) for the uncertaintified input fuzzy word are shown in Figure 14(a). Equation (6) is used for decisiveness before calculating COG for defuzzification.

As shown in Figure 14(a), the output is affected by injecting uncertainty to fuzzy input T1MF. Figure 14(b) shows that applied uncertainty makes the rules to be weakened according to the injected uncertainty to each point of input in the related fuzzy membership function. In this case, the rule is "if input is small then output is small," but if the effect of uncertainty is considered in Figure 14(b), it can be seen that the response after applying the uncertainty is greater than before and in points, where input data have a 
TABLE 1: 32 basic IT2FS words and their defuzzified output obtained by proposed equation (6) and their difference percentage compared to their COG calculated through the KM algorithm.

\begin{tabular}{|c|c|c|c|c|c|}
\hline & $\mathrm{UMF}$ & LMF & $\mathrm{KM}$ & DMF & Difference \\
\hline 1 & {$[0,0,0.14,1.97,1]$} & {$[0,0,0.05,0.66,1]$} & 0.47 & 0.459 & 0.01 \\
\hline 2 & {$[0,0,0.14,1.97,1]$} & {$[0,0,0.01,0.13,1]$} & 0.56 & 0.693 & -0.13 \\
\hline 3 & {$[0,0,0.26,2.63,1]$} & {$[0,0,0.05,0.63,1]$} & 0.63 & 0.634 & 0.004 \\
\hline 4 & {$[0,0,0.36,2.63,1]$} & {$[0,0,0.05,0.63,1]$} & 0.64 & 0.637 & 0.003 \\
\hline 5 & {$[0,0,0.64,2.47,1]$} & {$[0,0,0.10,1.16,1]$} & 0.66 & 0.610 & 0.05 \\
\hline 6 & {$[0,0,0.64,2.63,1]$} & {$[0,0,0.09,0.99,1]$} & 0.67 & 0.624 & 0.046 \\
\hline 7 & {$[0.59,1.50,2.00,3.41,1]$} & {$[0.79,1.68,1.68,2.21,0.74]$} & 1.75 & 1.702 & 0.048 \\
\hline 8 & {$[0.38,1.50,2.50,4.62,1]$} & {$[1.09,1.83,1.83,2.21,0.53]$} & 2.13 & 2.101 & 0.029 \\
\hline 9 & {$[0.09,1.25,2.50,4.62,1]$} & {$[1.67,1.92,1.92,2.21,0.30]$} & 2.19 & 2.360 & -0.17 \\
\hline 10 & {$[0.09,1.50,3.00,4.62,1]$} & {$[1.79,2.28,2.28,2.81,0.40]$} & 2.32 & 2.347 & -0.027 \\
\hline 11 & {$[0.59,2.00,3.25,4.41,1]$} & {$[2.29,2.70,2.70,3.21,0.42]$} & 2.59 & 2.601 & -0.011 \\
\hline 12 & {$[0.38,2.50,5.00,7.83,1]$} & {$[2.88,3.61,3.61,4.21,0.35]$} & 3.90 & 3.969 & -0.069 \\
\hline 13 & {$[1.17,3.50,5.50,7.83,1]$} & {$[4.09,4.65,4.65,5.41,0.40]$} & 4.56 & 4.605 & -0.045 \\
\hline 14 & {$[2.59,4.00,5.50,7.62,1]$} & {$[4.29,4.75,4.75,5.21,0.38]$} & 4.95 & 5.028 & -0.128 \\
\hline 15 & {$[2.17,4.25,6.00,7.83,1]$} & {$[4.79,5.29,5.29,6.02,0.41]$} & 5.13 & 5.162 & -0.032 \\
\hline 16 & {$[3.59,4.75,5.50,6.91,1]$} & {$[4.86,5.03,5.03,5.14,0.27]$} & 5.19 & 5.231 & -0.041 \\
\hline 17 & {$[3.59,4.75,6.00,7.41,1]$} & {$[4.79,5.30,5.30,5.71,0.42]$} & 5.41 & 5.398 & 0.012 \\
\hline 18 & {$[3.38,5.50,7.50,9.62,1]$} & {$[5.79,6.50,6.50,7.21,0.41]$} & 6.50 & 6.500 & 0.0 \\
\hline 19 & {$[4.38,6.50,8.00,9.41,1]$} & {$[6.79,7.38,7.38,8.21,0.49]$} & 7.16 & 7.183 & -0.023 \\
\hline 20 & {$[4.38,6.50,8.00,9.41,1]$} & {$[6.79,7.38,7.38,8.21,0.49]$} & 7.16 & 7.183 & -0.023 \\
\hline 21 & {$[4.38,6.50,8.25,9.62,1]$} & {$[7.19,7.58,7.58,8.21,0.37]$} & 7.25 & 7.212 & 0.038 \\
\hline 22 & {$[5.38,7.50,8.75,9.81,1]$} & {$[7.79,8.22,8.22,8.81,0.45]$} & 7.90 & 7.865 & -0.035 \\
\hline 23 & {$[5.38,7.50,8.75,9.83,1]$} & {$[7.69,8.19,8.19,8.81,0.47]$} & 7.91 & 7.874 & 0.036 \\
\hline 24 & {$[5.38,7.50,8.75,9.81,1]$} & {$[7.79,8.30,8.30,9.21,0.53]$} & 8.01 & 8.062 & -0.052 \\
\hline 25 & {$[5.38,7.50,9.00,9.81,1]$} & {$[8.29,8.56,8.56,9.21,0.38]$} & 8.03 & 7.97 & 0.06 \\
\hline 26 & {$[5.98,7.75,8.60,9.52,1]$} & {$[8.03,8.36,8.36,9.17,0.57]$} & 8.12 & 8.186 & -0.066 \\
\hline 27 & {$[7.37,9.41,10,10,1]$} & {$[8.72,9.91,10,10,1]$} & 9.30 & 9.344 & -0.044 \\
\hline 28 & {$[7.37,9.82,10,10,1]$} & {$[9.74,9.98,10,10,1]$} & 9.31 & 9.182 & 0.128 \\
\hline 29 & {$[7.37,9.59,10,10,1]$} & {$[8.95,9.93,10,10,1]$} & 9.34 & 9.372 & -0.032 \\
\hline 30 & {$[7.37,9.73,10,10,1]$} & {$[9.34,9.95,10,10,1]$} & 9.37 & 9.369 & 0.001 \\
\hline 31 & {$[7.37,9.82,10,10,1]$} & {$[9.37,9.95,10,10,1]$} & 9.38 & 9.368 & 0.012 \\
\hline \multirow[t]{2}{*}{32} & {$[8.68,9.91,10,10,1]$} & {$[9.61,9.97,10,10,1]$} & 9.69 & 9.693 & -0.003 \\
\hline & & Percentage of RMS of difference & & & $1.2 \%$ \\
\hline
\end{tabular}

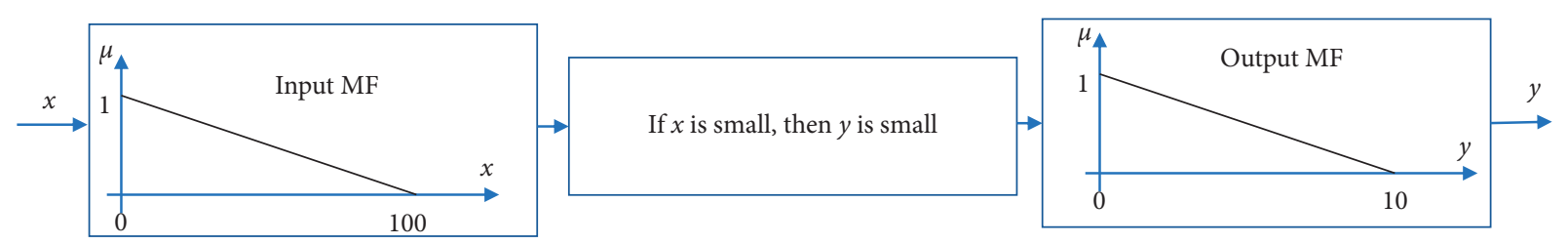

Figure 12: The single-input single-output single-rule fuzzy system. Rule: if $X$ is small, then $Y$ is small. Implication $=$ Min. $X$ (input); T1MF:

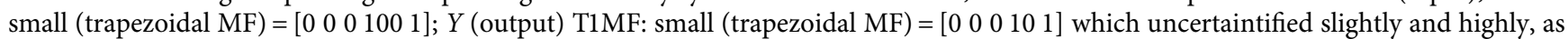
shown in Figures 13(a) and 13(c), respectively.

greater amount of uncertainty, and the output significantly becomes greater than before. It shows the concept of "small" in output to some extend is shifted towards the concept of "large" proper to the amount of uncertainty in correspondent point in the input.

Investigation 5: Uncertainty Avoidance and Noise

Usually data derived from industrial environments are contaminated with heavy noises [27-32]. If the uncertainty is caused by the noise, the behaviour of IT2FS is in such a way that the output of the system will be directed toward the less noisy data. The maximum displacement can be controlled by an appropriate definition of the decisive function. For $\left(\mu_{\text {decisive }}=\right.$ $\left.\mu_{\mathrm{p}}-(1 / 2) U^{2}\right)$, if the imbalance arrangement of the noise in the data (or any other uncertainty) is in the most imbalanced condition, the output will be displaced at maximum to the lownoise half of interval T2 fuzzy word. As Figure 15 shows, the half of the fuzzy word means the left side and right side.

Figure 15(a) shows a word which is the output of a system and the membership degree is 0.5 in all its points. All points are free of noise and uncertain. If the center of gravity is used for defuzzification, the center of gravity will be the point $y=a / 2$. Now, it is assumed that the inputs to the system are noisy so that the final derived fuzzy word is as shown in Figure 15(b), where the left side of the gravity 


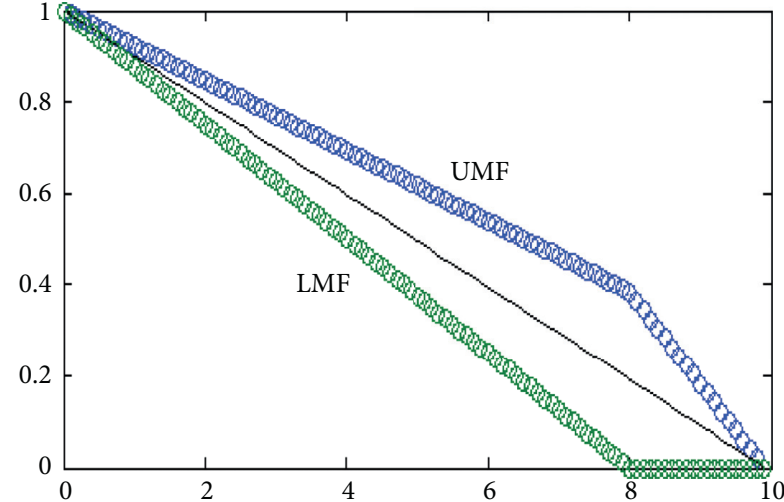

(a)

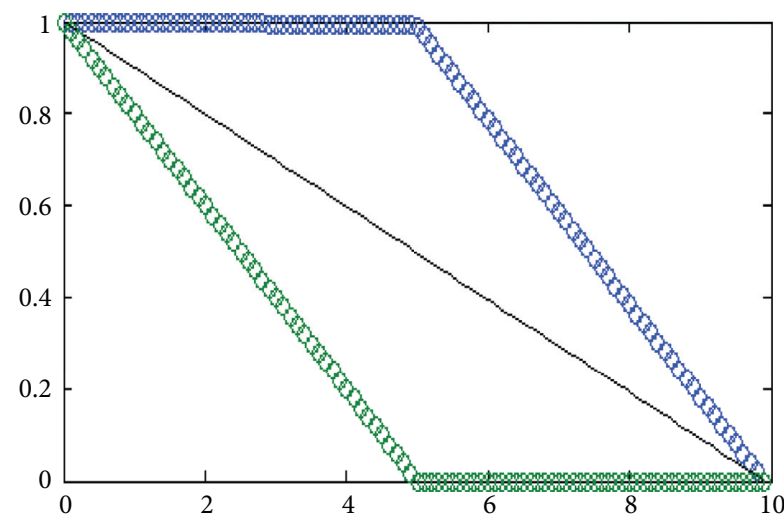

(c)

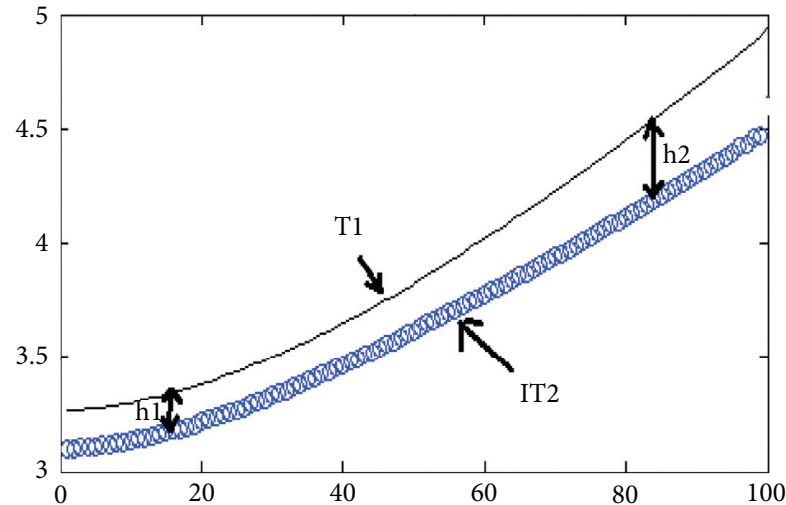

(b)

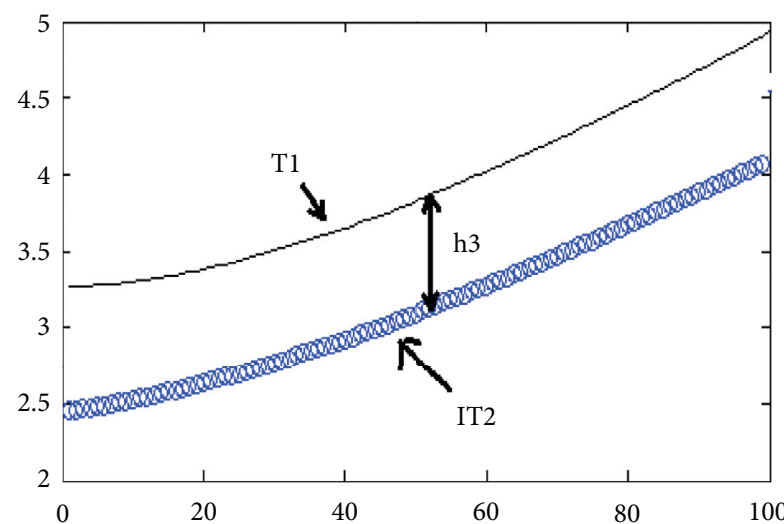

(d)

FIGURE 13: Effect of uncertaintified output fuzzy words on outputs of SISO: (a) output MF (small) slightly uncertaintified; (b) system output of (a); (c) output MF (small) highly uncertaintified; (d) system output of (a).

center is $100 \%$ noisy (totally uncertain). In this case, if equation (6) $\mu_{\text {decisive }}=\mu_{\mathrm{p}}-(1 / 2) U^{2}$ is used, the membership degree of all members in left side will be zero, and the new center is shifted to $y=a / 4$.

In fact, this point is the center of gravity of half of the membership function in Figure 15(c) as well. In Figure 15(c), it can be seen that the new output has the maximum possible distance from the previous center and the system response is shifted to a more certain region.

The reason may be that all input data and $y$ (i.e., the interval $[a / 2 a])$ involved in the fuzzy inference (the data involved in producing the output in a desired interval) to obtain the fuzzy weight (membership degree) of the output word in the interval $[a / 2 a]$ may be $100 \%$ noisy or, in other words, $100 \%$ uncertain.

However, the criterion $\mu_{\text {decisive }}=\mu_{\mathrm{p}}-(1 / 2) U^{2}$ for decisiveness to determine the output involves data based on the weight of uncertainty. This means that the decisiveness ignores the data completely uncertain or completely noisy and does not allow them to interfere with the output. So, as it can be seen in Figure 15, after injection of uncertainty, the output of $y=a / 4$ is formed only by data quite certain.

Investigation 6: Comparison between Collapsing Method and Decisiveness Method
The defuzzified outputs are calculated by using the collapsing method [10] and decisive equation (equation (6)):

(a) The MF of the upper and lower bands that are 0.8 and 0.2 in the range of the primary domain

(b) Symmetric triangle MF, UMF $=\left[\begin{array}{lllll}0.1 & 0.4 & 0.4 & 0.7 & 1\end{array}\right]$, and $\mathrm{LMF}=\left[\begin{array}{lllll}0.2 & 0.4 & 0.4 & 0.6 & 1\end{array}\right]$

(c) Asymmetric Gaussian MF, $\mathrm{UMF}=\left[\begin{array}{ll}0.3 & 0.2\end{array}\right]$, and $\mathrm{LMF}=\left[\begin{array}{ll}0.18 & 0.3\end{array}\right]$

Answer: in case of symmetric IT2MFs, the use of decisive equation (Equation (6)) causes no error. The error is zero when the upper and lower bounds are considered continuous functions or applied with few samples. The defuzzified outputs for the upper and lower bounds are 0.5 and 0.4 , respectively. The collapsing method with 11 or more samples is slightly different with the exact answer. For the asymmetric Gaussian IT2MF, the result obtained by decisive equation for 100 samples is 0.33 ; the result decreases with increasing number of samples. By using the continuous formula of the upper and lower MFs, the output converges to 0.3219 .

Investigation 7: Comparison between UW method [33] and Determiners Proposed in [25] 


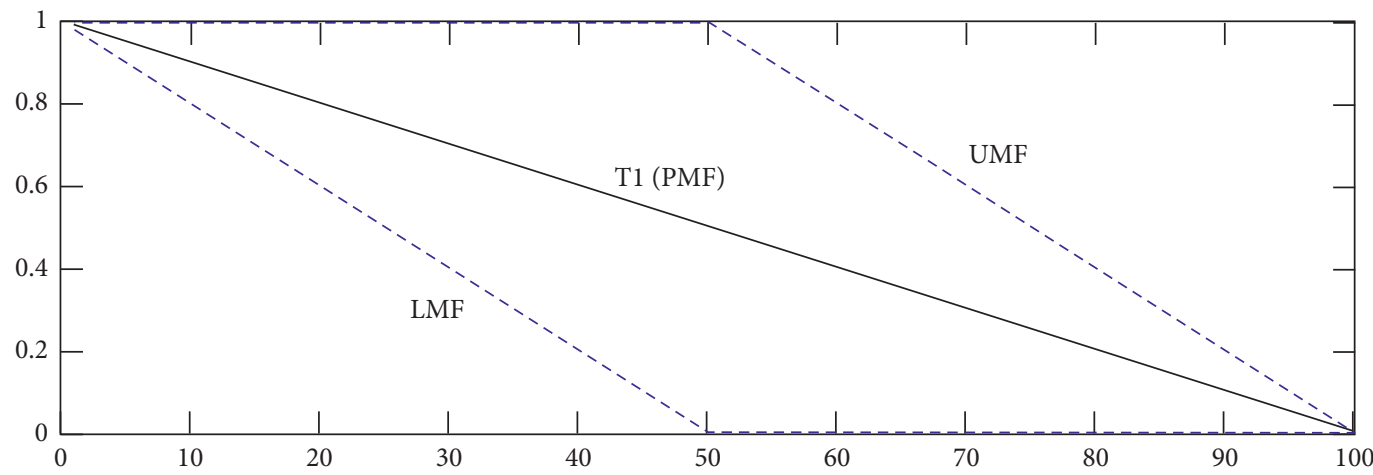

(a)

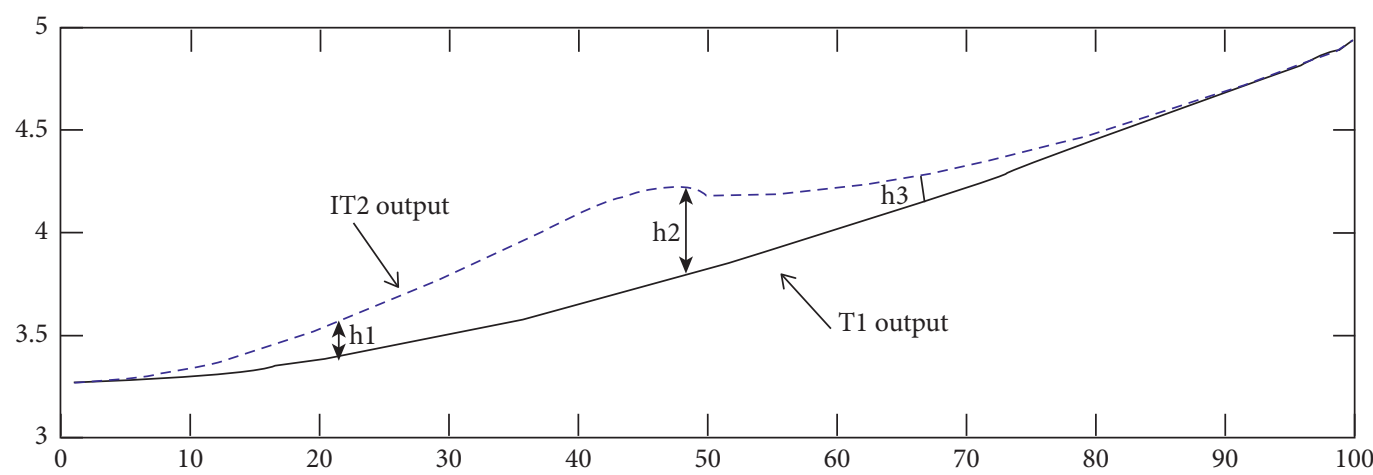

(b)

FIGURE 14: Effect of uncertaintified input fuzzy word on outputs of a SISO: (a) input membership function (small); (b) system output.

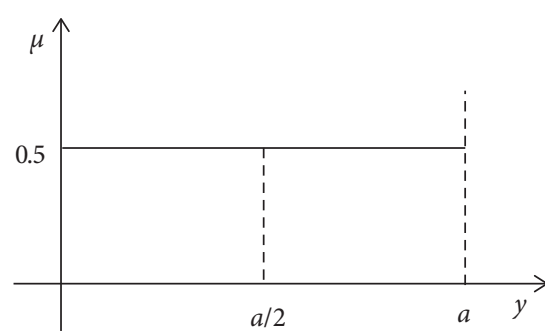

(a)

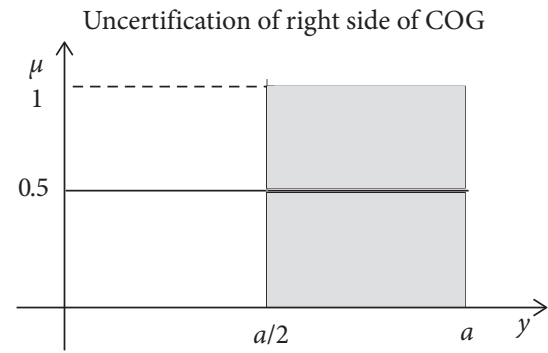

(b)

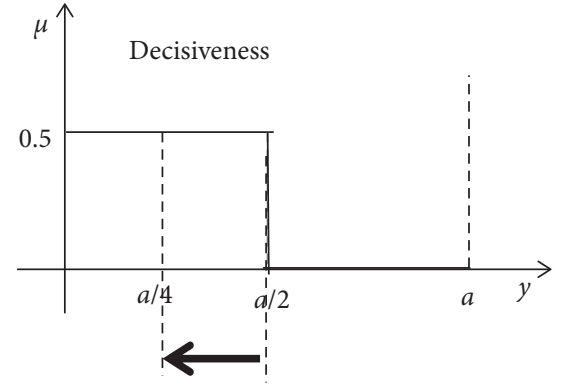

(c)

Figure 15: (a) Certain T1MF; (b) completely uncertaintified membership degrees of right side due to presence of noise in right side; (c) decisiveness formula applied before calculating the COG.

Runkler et al. [19] presented uncertainty weights method which makes a T1MF as the representative of IT2MF using $u(x)=1 / 2\left(\mu_{\mathrm{u}}(x)+\mu_{1}(x)\right)(1+\mu l(x)-\mu u(x))^{\alpha}$. This equation can be rewritten with parameters defined in [25] as $u(x)=\mu_{\mathrm{p}}(x) \times \beta$ in which $\mu_{\mathrm{p}}(x)=$ principal function membership degree and $\beta=(1+\mu l(x)-\mu u(x))^{\alpha}$ if $\alpha=1$ and $\beta=(1-\underline{\mathcal{u}})$ considering that $\left(\tilde{\mathcal{u}}=\mu_{\mathrm{u}}(x)-\mu_{\mathrm{l}}(x)\right) . u(x)=\mu_{\mathrm{p}} \times$ $(1-\underline{u})$, and then, $u(x)=\mu_{\mathrm{p}}-\left(\mu_{\mathrm{p}} \times \underset{u}{\mathcal{u}}\right)$; comparing with my equation (5), $U=1 / 2 \underset{\sim}{\mathcal{u}} \times \underset{\sim}{\mathcal{u}}$; in UW method, $U=\mu_{\mathrm{p}} \times \underset{\sim}{\mathcal{u}}$.

My proposed method works conceptually based on losing membership degree proportionally (not necessarily linearly) to uncertainty interval around PMF members. In
Runkler's, this uncertainty portion except to $\underset{\sim}{ }$ depends on $\mu_{\mathrm{p}}$ (considering $U=\mu_{\mathrm{p}} \times \underline{u}$ ). In this method, sometimes larger uncertainty weight $(\widetilde{u})$ decreases $\mu_{\mathrm{p}}$ equal to smaller uncertainty weight, due to different $\mu_{\mathrm{p}}$ values. This causes not to be in accordance with uncertainty avoidance and uncertainty handling perfectly.

If $\underset{\sim}{u}$ (uncertainty weight or uncertainty band) varies in [0 $1]$, then $\beta$ changes between $\left[\begin{array}{ll}1 & 0\end{array}\right]$ bounds. Therefore, $u(x)=\mu_{\mathrm{p}}(x) \times \beta$ decreases the $\mu_{\mathrm{p}}(x)$. But $u(x)$ does not vary between $\left[\mu_{1}(x) \mu_{\mathrm{p}}(x)\right]$ or between $\left[\mu_{\mathrm{p}}(x) \mu_{\mathrm{u}}(x)\right]$ to guarantee that $u(x)$ is an embedded MF between upper and lower bounds; Figure 16 confirms this claim. The Gaussian MFs 


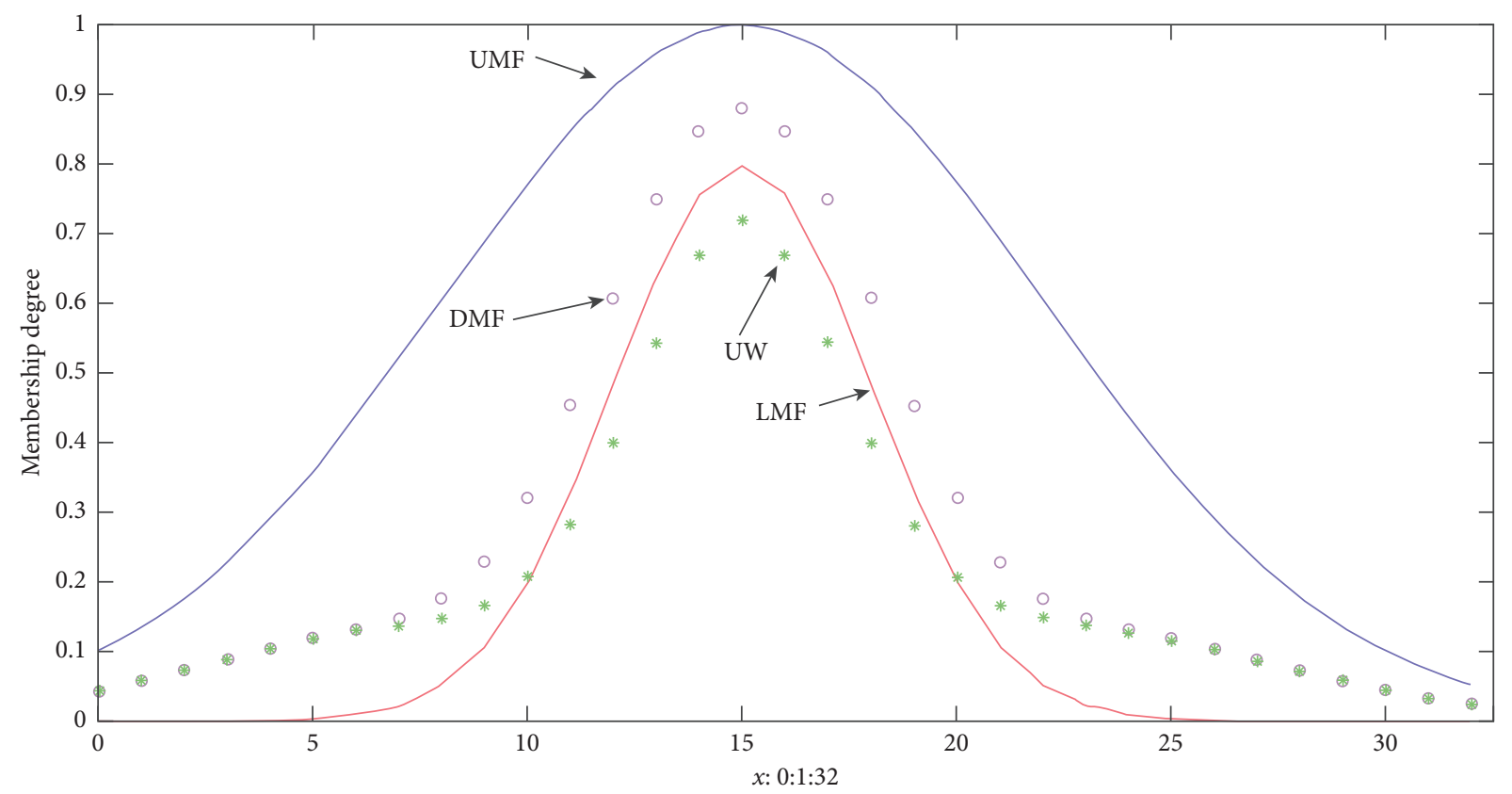

FIgURE 16: An example of UW method MF (denoted as *) and decisive method MF (denoted as o).

used in Figure 16 are upper MF $=\exp (-0.5 *(((x-15) / 7) 2))$ and lower $\mathrm{MF}=0.8 * \exp (-0.5 *(((x-15) / 3) 2))$.

Investigation 8: Uncertainty Effect on a Two-Input OneOutput IT2FS

Our proposed IT2FS that is used for this investigation has the below block diagram. A general MATLAB code is provided for two-input one-output uncertainty avoider IT2FS. Using this code is allowed for all researchers which work on it by citing this paper properly (MATLAB codes are indexed: Supplementary materials: Tables S1 and S2).

Inputs ( $x$ and $y$ ) and the output $(z)$ ranges are divided by four symmetrical Gaussian MFs with $50 \%$ overlap. Upper and lower MFs are chosen with the same center. The height of lower MFs is considered smaller than one. It is considered as T1MF, in case of equal heights for lower and upper MFs and equal to one. For having more uncertainty in a special $\mathrm{MF}$, the uncertainty band must increase. In other words, the height of lower MF must be chosen much smaller than one.

In this investigation, it is considered all inputs are T1MFs with no interval T2 uncertainty. "Low" and "medium minus" ranges of the output are considered as T1MF, but highly uncertain IT2MFs are chosen for "medium plus" and "high" ranges of the output. The 16 applied rules are shown in Table 2 .

The four edges of Figure 18(b) is uncertain because of uncertain "high" range in output $(z)$ as listed in Table 2 . The center part of Figure 18(b) remains unchanged compared to Figure 18(a), as shown in Figure 18(f). The value of uncertain part of Figure 18(b) decreases, as shown in Figure 18(f), as proved in investigation 3. And high values move toward center which is certain ( $\mathrm{T} 1$ is more certain than IT2) as it is clearly seen in Figures 18(c), 18(e), and 18(f).
TABLe 2: The 16 applied rules to the IT2FS illustrated in Figure 17.

\begin{tabular}{lcccc}
\hline $\begin{array}{l}x \text { and } y \\
\text { (inputs) }\end{array}$ & Low & $\begin{array}{c}\text { Medium } \\
\text { minus }\end{array}$ & $\begin{array}{c}\text { Medium } \\
\text { plus }\end{array}$ & High \\
\hline Low & $\begin{array}{l}\text { High } \\
(\text { IT2) }\end{array}$ & High (IT2) & High (IT2) & $\begin{array}{l}\text { High } \\
(\text { IT2) }\end{array}$ \\
$\begin{array}{l}\text { Medium } \\
\text { minus }\end{array}$ & $\begin{array}{l}\text { High } \\
\text { (IT2) }\end{array}$ & Low (T1) & Low (T1) & $\begin{array}{l}\text { High } \\
(\text { IT2 })\end{array}$ \\
Medium plus & $\begin{array}{l}\text { High } \\
\text { (IT2) }\end{array}$ & Low (T1) & Low (T1) & $\begin{array}{l}\text { High } \\
(\text { IT2) }\end{array}$ \\
High & $\begin{array}{l}\text { High } \\
\text { (IT2) }\end{array}$ & High (IT2) & High (IT2) & $\begin{array}{l}\text { High } \\
(\text { IT2) }\end{array}$ \\
\hline
\end{tabular}

4.2. Discussion. This argument is totally consistent with the principle of minimum uncertainty. The goal of minimum uncertainty principal is to ignore more uncertain data. In other words, the role of each data on decisions is reduced as much as the data are contaminated by uncertainties. The only point is that whether reducing the effect on output is always coincident with declining the membership degree in the uncertaintified MF. This research on the behaviour of IT2MF facing the uncertainties approves this issue; i.e., the membership degree is reduced due to the presence of uncertainty. In other words, IT2MF shows a conservative behaviour facing uncertainties.

But this is where the IT2MF imposes the problem. Generally, beyond adherence to IT2MF behaviour, increasing the degree of membership facing addition of uncertainty may reduce the effect of more uncertain data on the output. This behaviour is introduced as reckless logic versus conservative behaviour of IT2MF. However, it needs to be 


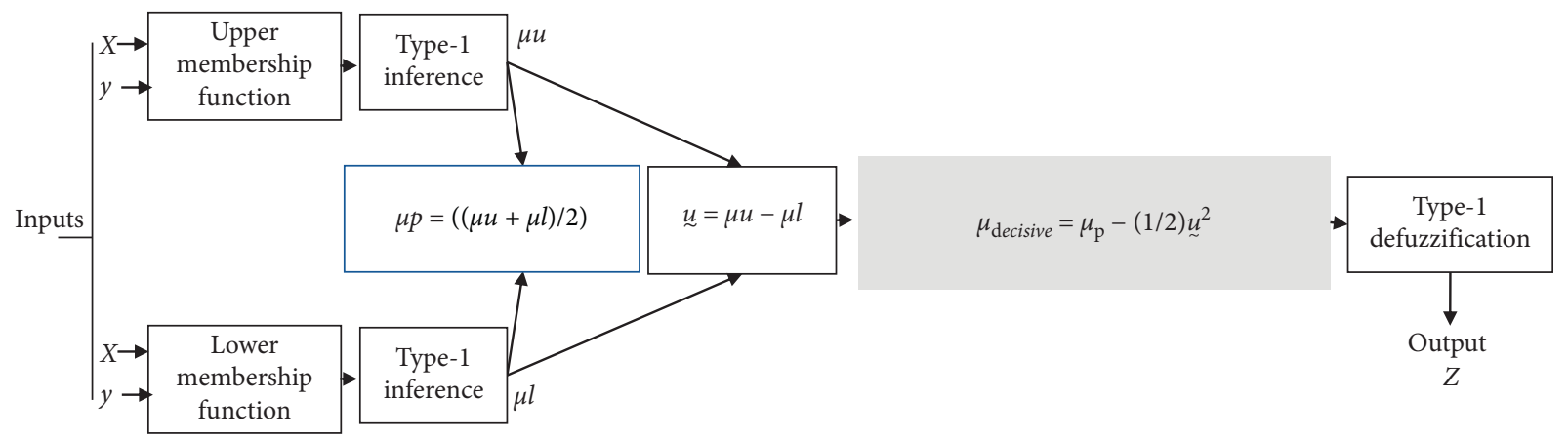

FIGURE 17: The structure of a two-input one-output IT2FS based on the decisiveness method.
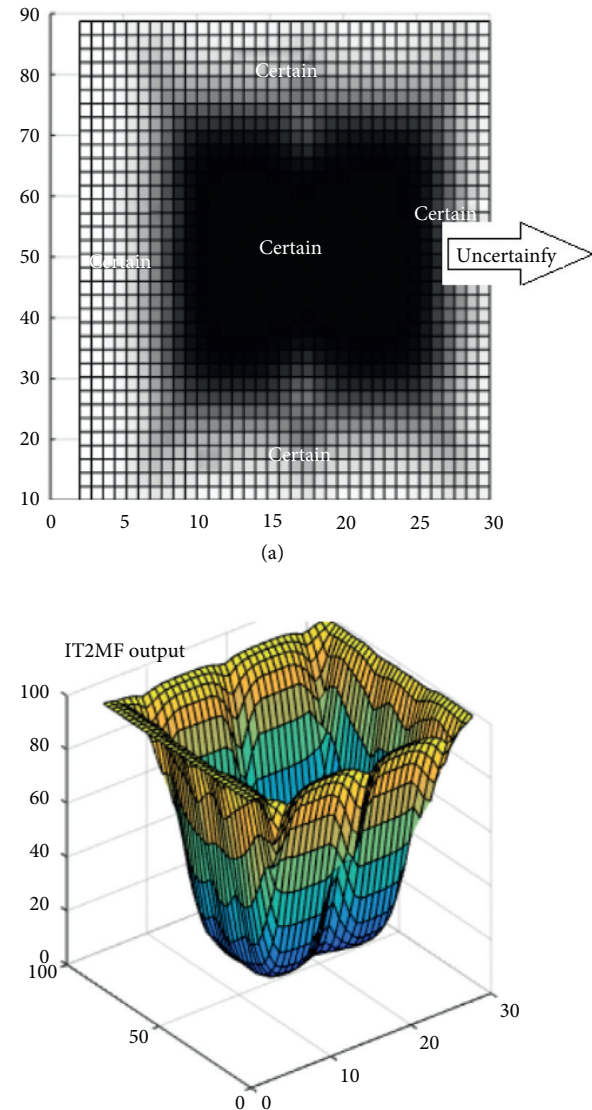

(d)
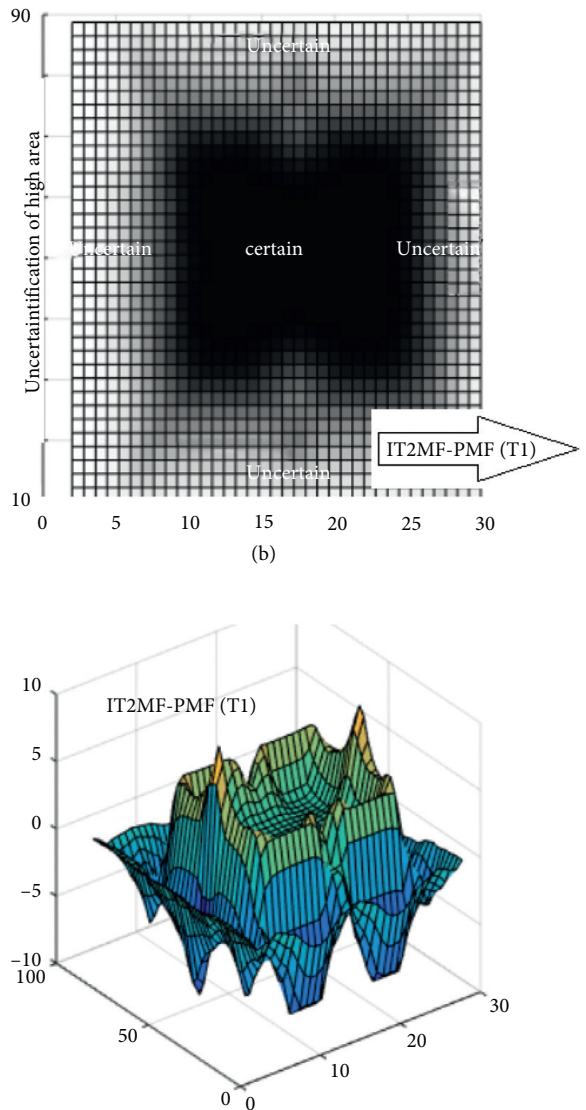

(e)
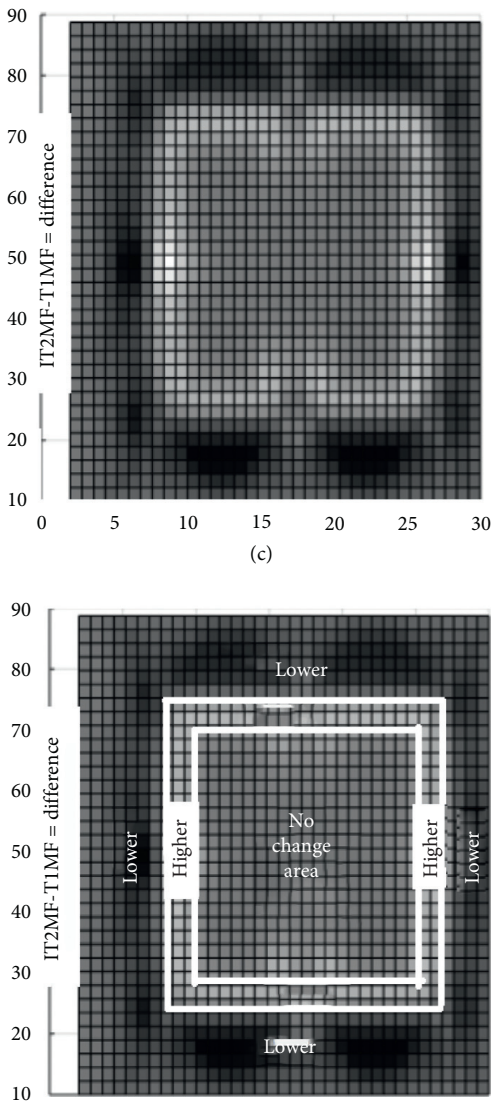

(f)

FIgURE 18: Output of two-input one-output IT2FS illustrated in Figure 16 with 16 rules of Table 2: (a) gray map of T1 PMF output; (b) uncertaintified T1 (IT2FMs); (c) difference between (a) and (b); (d) IT2MF 3D output; (e) 3D view of (c); (f) part (c) with more details.

investigated that which one of the existing systems shows such behaviour or when a system needs a reckless behaviour.

Equation (6) is a version of more common equation discussed in equation (9) in paper [20]. Therefore, common version of equation (6) is : $\mu_{\text {decisive }}=\mu_{\mathrm{p}}-(1 / 2 n) U^{\alpha}$, where $n$ and $a$ can intensify weakening of PMF due to presence of uncertainty. A prototype of this equation with $n=2$ and $\alpha=2$ is applied for interval type- 2 fuzzy c-means algorithm for color image segmentation successfully [34].

The proposed method eased calculation of defuzzification of IT2MF so that the results do not miss any main feature of IT2FSs. In case of slight output differences between the proposed method and KM algorithm, there is no mathematic proof which shows the outputs gained by the $\mathrm{KM}$ algorithm are absolutely desired. On the contrary, due to the existence of concepts and reasons behind the proposed method, it provides a designer with more opportunities to manage parameters related to uncertainty in T2FSs conveniently in engineering and industrial applications more conveniently and up-to-date.

An important thing for a designer who works with fuzzy systems is that he/she must have information about the behaviour of used fuzzifier, inference, and defuzzifier methods in the system. There is no defuzzification method 
which is most suitable in all systems and all conditions. If method A releases better results than method $B$ in some conditions, it is probable that $\mathrm{B}$ is better than $\mathrm{A}$, if condition or system changes. For choosing appropriate method, the experience of designer plays the main role. In case of this research, understanding and absorbing of system behaviour is easier.

Considering uncertainty avoidance as a basic nature of IT2FSs (or basic IT2FSs expectation to differ from T1FSs), some proposed defuzzification methods such as Begian et al. [5], Du and Ying [6], Nie and Tan [7], or proposed system equivalents such as [35] are easily excluded from IT2FS category.

Considering presented investigations, it can be said that the uncertainty reduces the value of membership degrees and the absolute value of words. Higher uncertainty causes higher reduction of values. For example, if in input and output of a system, there exist the words "Low, Medium, and Large" and then after injecting uncertainty to inputs, output "small" will be shifted towards "Medium," "Medium" towards "Large," and "Large" towards "Medium." The results show that "the uncertainty reduces the membership degree value, proportionally, and the uncertainty in inputs of system shifts the concept of words towards the opposite words in output of the system, proportionally."

The proposed method is more conceptual and simpler than the Greenfield-Chiclana collapsing method, which uses an iterative procedure proposed in Greenfield et al. [10]. Our proposed method clearly describes the effect of different levels of noise by using the proposed formula, as shown in investigation 5. Others investigate noise effect using experiment method and consider T2 fuzzy system as a block box for example [36]. Straight and simple formulas are proposed for defuzzification, and the resulting formulas show clearly the relationship between amount of uncertainty and resultant collapsed value. Because of that, managing system design with the proposed formula is easier and more conceptual.

Obviously, the introduced general principles of the proposed uncertainty avoidance method are capable of being generalised to other systems dealing with uncertainty and their DMFs (decisive membership functions) will be defined based on the behaviour of those systems A look-up table data driven based method is presented in [32] for extracting the rules of an IT2FS based on uncertainty avoider.

\section{Conclusion}

The results of this research include providing a new look at the relationship between uncertainty and defuzzification, introducing new criteria for recognising correct defuzzification methods in the interval T2FSs, presenting new defuzzification method based on decisiveness of fuzzy words, and studying the IT2FSs through the uncertainty removal issue. For each T1MF, it is interpreted as uncertaintified functions. In addition, for each IT2MF function, a decisive function was defined and its extraction method was explained. While presenting a general concept of decisiveness, a decisive MF was presented, which was based on finding IT2MF center of gravity or the points close to it.

It is proved that using symmetric and similar MFs is not always effective and meaningful. For handling uncertainty, replacing IT2MF with T1MF and choosing the uncertainty band of IT2Ms must be done based on the rate of presence of uncertainty in corresponding available input and output data for handling uncertainty in a desired fuzzy system.

The proposed method can be considered as a closed formula for calculating IT2MF defuzzification which works on the basis of uncertainty avoidance. Decisive membership function $\left(\mu_{\text {decisive }}=\mu_{\mathrm{p}}-(1 / 2) U^{2}\right)$ provides a simple, quick, and conceptual method for defuzzification of IT2MF. While losing none of the advantages of the interval T2FSs through using this method, it requires fewer calculations than several algorithms available for calculating output of the interval T2FSs. This method has greater ability to be implemented on specific hardware applications. It is easily extendable for uncertain singleton fuzzy outputs, as well. Besides, due to the existence of concepts and reasons behind the proposed method, it provides a designer with more opportunities to manage parameters related to uncertainty in IT2FSs conveniently in engineering and industrial applications more convenient and more informed.

\section{Data Availability}

All codes and data used are available upon request.

\section{Conflicts of Interest}

The author declares that there are no conflicts of interest.

\section{Supplementary Materials}

This supplementary material includes a MATLAB code for a two_input one_output Uncertainty Avoider based Interval Type II Fuzzy System. The program includes fuzzifier, inference, uncertainty removing (Decisiveness block) and defuzzifier blocks. Four Membership functions are provided for inputs and output which are extendable. (Supplementary Materials)

\section{References}

[1] N. N. Karnik and J. M. Mendel, Uncertain Rule-Based Fuzzy Logic Systems: Introduction and New Directions, PrenticeHall, Upper-Saddle River, NJ, USA, 2001.

[2] S. Greenfield, F. Chiclana, R. John, and S. Coupland, "The sampling method of defuzzification for type-2 fuzzy sets: experimental evaluation," Information Sciences, vol. 189, pp. 77-92, 2012.

[3] J. M. Mendel and X. Liu, "New closed-form solutions for Karnik-Mendel algorithm+ defuzzification of an interval type-2 fuzzy set," in Proceedings of the 2012 IEEE International Conference on Fuzzy Systems, pp. 1-8, IEEE, Brisbane, Australia, June 2012.

[4] H. Wu and J. M. Mendel, "Uncertainty bounds and their use in the design of interval type-2 fuzzy logic systems," IEEE Transactions on Fuzzy Systems, vol. 10, no. 5, pp. 622-639, 2002, p. 
[5] M. B. Begian, W. W. Melek, and J. M. Mendel, "Parametric design of stable type-2 TSK fuzzy systems," in Proceedings of the NAFIPS 2008-2008 Annual Meeting of the North American Fuzzy Information Processing Society, pp. 1-6, IEEE, New York City, NY, USA, May 2008.

[6] X. Du and H. Ying, "Derivation and analysis of the analytical structures of the interval type-2 fuzzy-PI and PD controllers," IEEE Transactions on Fuzzy Systems, vol. 18, no. 4, pp. 802814, 2010, p.

[7] M. Nie and W. W. Tan, "Towards an efficient type-reduction method for interval type- 2 fuzzy logic systems," $p$, IEEE, in Proceedings of the 2008 IEEE International Conference on Fuzzy Systems (IEEE World Congress on Computational Intelligence), pp. 1425-1432, IEEE, Hong Kong, China, June 2008.

[8] R. John and S. Coupland, "Type-2 fuzzy logic: a historical view," IEEE Computational Intelligence Magazine, vol. 2, no. 1 , pp. 57-62, 2007.

[9] D. Wu and W. W. Tan, "Computationally efficient type-reduction strategies for a type-2 fuzzy logic controller," in Proceedings of the the 14th IEEE International Conference on Fuzzy Systems, 2005. FUZZ'05, pp. 353-358, IEEE, Reno, NV, USA, May 2005.

[10] S. Greenfield, F. Chiclana, S. Coupland, and R. John, "The collapsing method of defuzzification for discretised interval type-2 fuzzy sets," Information Sciences, vol. 179, no. 13, pp. 2055-2069, 2009.

[11] D. Wu and J. M. Mendel, "A comparative study of ranking methods, similarity measures and uncertainty measures for interval type-2 fuzzy sets," Information Sciences, vol. 179, no. 8, pp. 1169-1192, 2009.

[12] $\mathrm{D}$. Wu, "On the fundamental differences between interval type-2 and type-1 fuzzy logic controllers," IEEE Transactions on Fuzzy Systems, vol. 20, no. 5, pp. 832-848, 2012.

[13] S. Aminifar, A. Khoei, K. Haidi, and G. Yosefi, "A digital CMOS fuzzy logic controller chip using new fuzzifier and max circuit," AEU-International Journal of Electronics and Communications, vol. 60, no. 8, pp. 557-566, 2006.

[14] O. Castillo, L. Cervantes, J. Soria, M. Sanchez, and J. R. Castro, "A generalized type-2 fuzzy granular approach with applications to aerospace," Information Sciences, vol. 354, pp. 165-177, 2016.

[15] L. Cervantes and O. Castillo, "Type-2 fuzzy logic aggregation of multiple fuzzy controllers for airplane flight control," Information Sciences, vol. 324, pp. 247-256, 2015.

[16] M. A. Sanchez, O. Castillo, and J. R. Castro, "Generalized type-2 fuzzy systems for controlling a mobile robot and a performance comparison with interval type- 2 and type- 1 fuzzy systems," Expert Systems with Applications, vol. 42, no. 14, pp. 5904-5914, 2015.

[17] C. E. Celemin and M. A. Melgarejo, "A proposal to speed up the computation of the centroid of an interval type-2 fuzzy set," Advances in Fuzzy Systems, vol. 2013, Article ID 158969, 19 pages, 2013.

[18] D. Wu and J. M. Mendel, "On the continuity of type-1 and interval type-2 fuzzy logic systems," IEEE Transactions on Fuzzy Systems, vol. 19, no. 1, pp. 179-192, 2010.

[19] T. A. Runkler, C. Chen, and R. John, "Type reduction operators for interval type-2 defuzzification," Information Sciences, vol. 467, pp. 464-476, 2018.

[20] S. Aminifar and A. bin Marzuki, "Horizontal and vertical rule bases method in fuzzy controllers," Mathematical Problems in Engineering, vol. 2013, Article ID 532046, 9 pages, 2013.

[21] P. Melin, E. Ontiveros-Robles, C. I. Gonzalez, J. R. Castro, and O. Castillo, "An approach for parameterized shadowed type-2 fuzzy membership functions applied in control applications," Soft Computing, vol. 23, no. 11, pp. 3887-3901, 2019.

[22] E. Ontiveros-Robles, P. Melin, and O. Castillo, "New methodology to approximate type-reduction based on a continuous root-finding karnik mendel algorithm," Algorithms, vol. 10, no. 3, p. 77, 2017.

[23] E. Ontiveros, P. Melin, and O. Castillo, "High order $\alpha$-planes integration: a new approach to computational cost reduction of general type-2 fuzzy systems," Engineering Applications of Artificial Intelligence, vol. 74, pp. 186-197, 2018.

[24] S. Aminifar, "Design and implementation of fuzzy controllers for handling uncertainty in an industrial application," $p$, Universiti Sains Malaysia, Penang, Malaysia, Doctoral dissertation, 2014.

[25] S. Aminifar and A. Marzuki, "Uncertainty in interval type-2 fuzzy systems," Mathematical Problems in Engineering, vol. 2013, Article ID 452780, 16 pages, 2013.

[26] J. Qin, X. Liu, and W. Pedrycz, "An extended TODIM multicriteria group decision making method for green supplier selection in interval type-2 fuzzy environment," European Journal of Operational Research, vol. 258, no. 2, pp. 626-638, 2017.

[27] S. Aminifar and A. B. Marzuki, "Voltage-mode fuzzy logic controller," Indian Journal of Science and Technology, vol. 5, no. 11, pp. 3630-3636, 2012.

[28] S. Aminifar and A. bin Marzuki, "A novel fuzzy controller applicable to steaming room," in Proceedings of the 2012 IEEE International Conference on Control System, Computing and Engineering, pp. 171-176, IEEE, Penang, Malaysia, November 2012.

[29] S. Aminifar and G. Yosefi, "Application of adaptive neuro fuzzy inference system (ANFIS) in implementing of new CMOS fuzzy logic controller (FLC) chip," in Proceedings of the AIP Conference Proceedings, vol. 936, no. 1, pp. 49-53, American Institute of Physics, 2007.

[30] A. Marzuki, S. Y. Tee, and S. Aminifar, "Study of fuzzy systems with Sugeno and Mamdani-type fuzzy inference systems for determination of heartbeat cases on electrocardiogram (ECG) signals," International Journal of Biomedical Engineering and Technology, vol. 14, no. 3, pp. 243-276, 2014.

[31] G. Yosefi and S. Aminifar, "Implementation of a CMOS mixed-signal digital FLC chip using new fuzzifier and current mode A/D," in Proceedings of the 2007 6th International Conference on Information, Communications \& Signal Processing, pp. 1-5, IEEE, Singapore, December 2007.

[32] S. M. KekShar and S. A. Aminifar, "Lookup table driven uncertainty avoider based interval type-2 Fuzzy system design," IEEE-SEM, vol. 8, no. 4, 2020.

[33] T. A. Runkler, S. Coupland, R. John, and C. Chen, "Interval type-2 defuzzification using uncertainty weights," in Frontiers in Computational Intelligence, pp. 47-59, Springer, Cham, Switzerland, 2018.

[34] A. Hamad, S. Aminifar, and M. Daneshwar, "An interval type2 FCM for color image segmentation," International Journal of Advanced Computer Research, vol. 10, no. 46, pp. 12-17, 2020.

[35] M. Khosla, R. K. Sarin, M. Uddin, S. Singh, and A. Khosla, "Realizing interval type-2 fuzzy systems with type-1 fuzzy systems," in Cross-Disciplinary Applications of Artificial Intelligence and Pattern Recognition: Advancing Technologies, pp. 412-427, IGI Global, Pennsylvania, PA, USA, 2012.

[36] E. Ontiveros-Robles, P. Melin, and O. Castillo, "Comparative analysis of noise robustness of type 2 fuzzy logic controllers," Kybernetika, vol. 54, no. 1, pp. 175-201, 2018 b. 